\title{
Direct numerical simulation of flow around a surface-mounted finite square cylinder at low Reynolds numbers
}

Di Zhang, Liang Cheng, Hongwei An, and Ming Zhao

Citation: Physics of Fluids 29, 045101 (2017); doi: 10.1063/1.4979479

View online: https://doi.org/10.1063/1.4979479

View Table of Contents: http://aip.scitation.org/toc/phf/29/4

Published by the American Institute of Physics

\section{Articles you may be interested in}

Free locomotion of a flexible plate near the ground

Physics of Fluids 29, 041903 (2017); 10.1063/1.4981778

Streamwise vortex breakdown in supersonic flows

Physics of Fluids 29, 054102 (2017); 10.1063/1.4982901

From two-dimensional to three-dimensional turbulence through two-dimensional three-component flows Physics of Fluids 29, 111101 (2017); 10.1063/1.4990082

The lowest Reynolds number of vortex-induced vibrations

Physics of Fluids 29, 041701 (2017); 10.1063/1.4979966

Direct numerical simulation of turbulent boundary layer with fully resolved particles at low volume fraction Physics of Fluids 29, 053301 (2017); 10.1063/1.4982233

Hairpin vortex organization in wall turbulence

Physics of Fluids 19, 041301 (2007); 10.1063/1.2717527

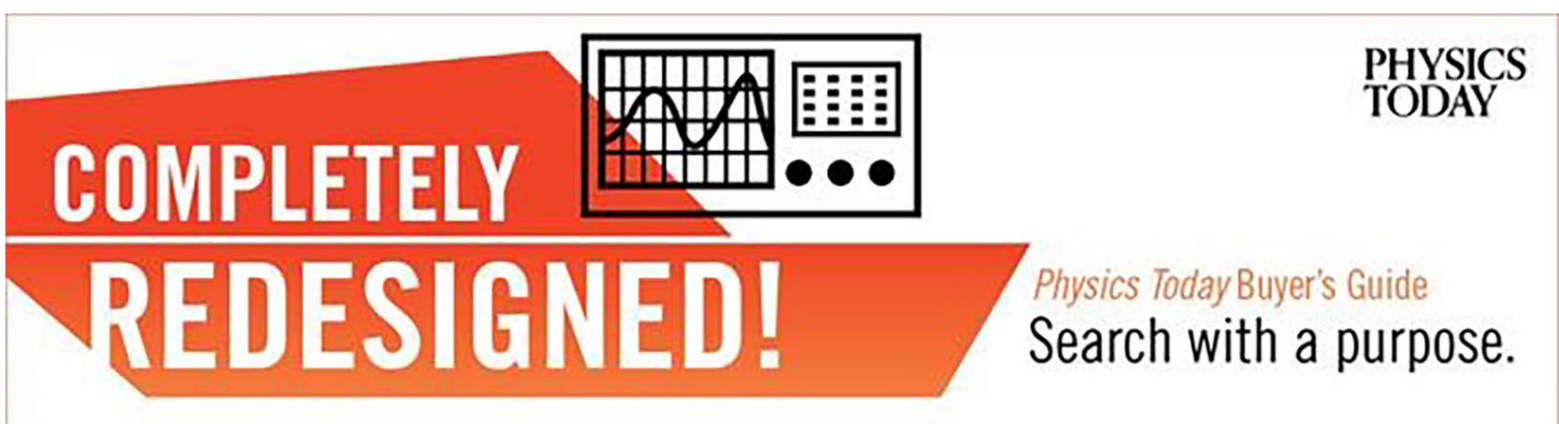




\title{
Direct numerical simulation of flow around a surface-mounted finite square cylinder at low Reynolds numbers
}

\author{
Di Zhang, ${ }^{1, a)}$ Liang Cheng, ${ }^{1,2, a)}$ Hongwei An, ${ }^{1}$ and Ming Zhao ${ }^{3}$ \\ ${ }^{1}$ School of Civil, Environmental and Mining Engineering, The University of Western Australia, \\ 35 Stirling Highway, Crawley, WA 6009, Australia \\ ${ }^{2}$ DUT-UWA Joint Research Centre, State Key Laboratory of Coastal and Offshore Engineering, \\ Dalian University of Technology, Dalian 116024, China \\ ${ }^{3}$ School of Computing, Engineering and Mathematics, Western Sydney University, Locked Bay 1797, \\ Penrith, NSW 2751, Australia
}

(Received 2 October 2016; accepted 16 March 2017; published online 5 April 2017)

\begin{abstract}
With the aid of direct numerical simulation, this paper presents a detailed investigation on the flow around a finite square cylinder at a fixed aspect ratio $(A R)$ of 4 and six Reynolds numbers $(R e=50$, $100,150,250,500$, and 1000). It is found that the mean streamwise vortex structure is also affected by $R e$, apart from the $A R$ value. Three types of mean streamwise vortices have been identified and analyzed in detail, namely, "Quadrupole Type" at $R e=50$ and $R e=100$, "Six-Vortices Type" at $R e=150$ and $R e=250$, and "Dipole Type" at $R e=500$ and $R e=1000$. It is the first time that the "Six-Vortices Type" mean streamwise vortices are reported, which is considered as a transitional structure between the other two types. Besides, three kinds of spanwise vortex-shedding models have been observed in this study, namely, "Hairpin Vortex Model" at $R e=150$, "C and Reverse- $C$ and Hairpin Vortex Model (Symmetric Shedding)" at $R e=250$, and " $C$ and Reverse-C and Hairpin Vortex Model (Symmetric/Antisymmetric Shedding)" at $R e=500$ and $R e=1000$. The newly proposed " $C$ and Reverse-C and Hairpin Vortex Model" shares some similarities with "Wang's Model" [H. F. Wang and Y. Zhou, "The finite-length square cylinder near wake," J. Fluid Mech. 638, 453-490 (2009)] but differs in aspects such as the absence of the connection line near the free-end and the " $C$-Shape" vortex structure in the early stage of the formation of the spanwise vortex. Published by AIP Publishing. [http://dx.doi.org/10.1063/1.4979479]
\end{abstract}

\section{INTRODUCTION}

Three-dimensional flow structures around a bottommounted cylinder have received considerable attention over the past decades, mainly due to their relevance in nature and engineering applications. ${ }^{1-6}$ Many earlier studies focused on the analysis of the flow past infinite two-dimensional (2D) circular or square cylinders. ${ }^{7-11}$ Recently, increasing attention has been paid to unsteady flow around finite-height cylinders, ${ }^{1-6,12-17}$ with one end immersed in the free stream (viz., the free end) and the other end mounted on a plane wall (viz., the base end). Due to the combined influence of the downwash flow from the free end, the horizontal streaming around the cylinder, and the boundary layer near the bottom wall, the three-dimensional flow structure around a finite-height cylinder placed on a nonslip surface is usually more complicated than that around a cylinder of infinite height.

It is understood from the literature that the following six factors influence the flow structure around a finite-height cylinder: ${ }^{1-4,15-25}$ (1) the turbulence intensity of the approaching flow; (2) the cross-sectional shape of the cylinder; (3) the Re number defined as $R e=U D / v$, where $D$ is the characteristic width of the cylinder, such as the diameter of a circular cylinder or the side-length of a square cylinder, $U$ is the free

a)Electronic addresses: di.zhang@uwa.edu.au and liang.cheng@uwa.edu.au. stream velocity, and $v$ is the fluid's kinematic viscosity; (4) the boundary-layer thickness on the bottom wall relative to the cylinder height (viz., $\delta / h)$; (5) the ratio of the cylinder height to the characteristic width of the cylinder (viz., $A R=h / D$ ); and (6) the blockage ratio of the channel (viz., $\beta_{1}=h / H$ and $\beta_{2}=D / B$, where $H$ and $B$ are the height and width of the channel or the computational domain, respectively). The relative importance of the aforementioned factors may vary across different scenarios. ${ }^{3,26-29}$ So far, most of the existing studies address the influence of only one or two factors, and, therefore, the integrated effect of several parameters is still not fully understood. ${ }^{2,3}$

Some interesting flow phenomena have been revealed by previous studies, such as the $U$-shaped horseshoe vortex system originating in front of a cylinder and extending downstream from either side of the cylinder; the flow separation from the leading edge of the top and side faces of the cylinder; the downward-directed flow from the top of the cylinder (viz., the downwash) and the recirculation region in the near-wake behind the cylinder. Many aspects of the horseshoe vortex, including the formation mechanism, the number of vortex cores, the flow regime (i.e., steady, periodic, or irregular), and the circulation and position of the primary vortex, have been extensively studied and well understood, as demonstrated by Baker, ${ }^{30,31}$ Lin et al. ${ }^{32}$ Pattenden et al.,${ }^{33}$ Hwang and Yang, ${ }^{18}$ Seal et al.,${ }^{34}$ Thomas, ${ }^{35}$ Ballio et al.,${ }^{36}$ and so on. However, open questions still exist for the complex 3D 
wake flow, which involves interactions between various types of vortices (i.e., tip vortices, spanwise vortices, base vortices, and horseshoe vortex legs), ${ }^{2,3,20,21,27,37}$ as discussed in the following paragraphs.

First of all, the streamwise vortex structure, as one of the most remarkable properties of the wake topology, still needs to be further explored. By conducting flow visualization experiments for two finite circular cylinders (i.e., $A R=1.25$ and 4.25) at two Reynolds numbers (i.e., $R e=5.92 \times 10^{3}$ and 1.48 $\times 10^{5}$ ), Roh and Park ${ }^{38}$ reported the formation and evolution of two pairs of counter-rotating "streamwise instantaneous tip vortices," namely, the tornado-like tip vortices (evolving from two spiral nodes on the top face) and the trailing side tip vortices (rooting from the circumferential edge of the free end). There have been different opinions about the number and the origin of tip vortices. For example, Kawamura et al. ${ }^{4}$ ( $A R$ $\left.=1-8, R e=3.2 \times 10^{4}\right)$, Park and Lee ${ }^{17,39}(A R=6-13$, Re $\left.=2 \times 10^{4}\right)$, Krajnovic ${ }^{5}\left(A R=6, R e=2 \times 10^{4}\right)$, Pattenden et al. ${ }^{33}\left(A R=1, R e=2 \times 10^{5}\right)$, Hain et al. ${ }^{40}(A R \approx 2.17$, Re $\left.=1 \times 10^{5}\right), \operatorname{Leder}^{41}\left(A R=2, R e=2 \times 10^{5}\right)$, and Johnston and Wilson ${ }^{42}$ found only one pair of "streamwise instantaneous tip vortices" near the free end. Transverse instantaneous tip vortex (being different from the streamwise tip vortex), caused by the vortex-shedding from the leading edge of the free end, has been observed by Saeedi et al. ${ }^{12}\left(A R=4, R e=1.2 \times 10^{4}\right)$ and Hwang and Yang ${ }^{18}(A R=1, R e=3500)$. Additionally, Sumner and Heseltine ${ }^{2}$ and Sumner et al. ${ }^{21}$ studied the time-averaged streamwise vortices in the wake of a finite circular cylinder $(A R$ $=3-9, R e=6 \times 10^{4}, \delta / D=2.6$ ), and their research showed that either a dipole type or a quadrupole type appears depending on the aspect ratio $(A R)$ value. At $A R=3$ (i.e., a dipole type), only a counter-rotating pair of mean streamwise tip vortices was identified at various streamwise sections in the wake, which was attributed to the downwash flow from the free end. For $A R \geq 5$ (i.e., a quadrupole type), besides the mean streamwise tip vortices, a pair of mean streamwise base vortices (with an opposite rotation direction to the tip ones) was observed within the turbulent boundary layer, which can be explained by the upwash flow near the bottom wall in the wake. Actually, this phenomenon has also been observed by Wang and Zhou, ${ }^{1}$ Pattenden et al. ${ }^{33}$ Porteous et al.,${ }^{27}$ Sumner, ${ }^{43}$ Frederich et al., ${ }^{44}$ and Bourgeois et al., ${ }^{3,45}$ who showed that the strength of mean streamwise base vortices was enhanced with the increase of thickness of the boundary layer (viz., $\delta / D$ ). ${ }^{25}$ Although the influence of the $A R$ value on the mean streamwise vortex structure has been thoroughly analyzed, the effect of the Re number on the types of the mean streamwise vortices and the transition process of vortices between the near and far wakes have rarely been discussed previously, which is one of the motivations of this study.

Furthermore, the variation of the vortex-shedding characteristics along the cylinder span has not been completely ascertained, mainly due to the 3D nature of the vortex structure and the diversity of the influencing factors. Okamoto and Sunabashiri ${ }^{24}\left(A R=0.5-7, R e=2.5 \times 10^{4}\right.$ to $\left.4.7 \times 10^{4}\right)$, Taniguchi et al. ${ }^{46}\left(A R=0.75-5, R e=9 \times 10^{3}\right.$ to $\left.7.2 \times 10^{4}\right)$, and Sakamoto and $\mathrm{Arie}^{23}(A R=0.5-5, R e=270-920)$ showed the existence of a critical aspect ratio at $A R_{C}=2-5$ which marks the change of vortex-shedding form, and the value of $A R_{c}$ is mainly affected by $R e, \delta / D$ and the cross-sectional shape of the cylinder. For $A R>A R_{c}$, the asymmetric Karman vortex street was observed in the wake because the vortex-shedding is dominated by the lateral free shear layers. ${ }^{2,47}$ For $A R<A R_{c}$, the flow near the free-end suppresses the Karman vortex and eventually results in the symmetric arch-type vortex along the span in the near wake. However, Kawamura et al. ${ }^{4}$ doubted the possibility of the formation of an arch-shaped vortex for low $A R$ values because of the existence of the trailing vortices. In addition, Wang et al. ${ }^{25}\left(A R=5, R e=1.15 \times 10^{4}\right)$ and Wang and Zhou ${ }^{1}$ $(A R=3-7, R e=9300)$ argued that, whether $A R$ is larger or smaller than the critical value, the anti-symmetric and symmetric vortex-shedding occur intermittently in various horizontal planes. The probability of occurrence of anti-symmetric and symmetric vortex-shedding along the cylinder span is dependent on the $A R, R e$, and $\delta / D$ values. Wang and Zhou ${ }^{1}$ proposed an empirical 3D vortex-shedding model (combining the tip, base, and spanwise vortices) around a finite square cylinder, in which the vortex filaments near the tip and near the base are bent upstream (caused by the downwash and upwash, respectively). With the increase of the distance from the cylinder, the bottom vortex filament becomes less inclined as a result of the decrease of the base-vortex circulation. This makes it possible that a quadrupole type in the near-wake transforms into a dipole type in further downstream regions in terms of mean streamwise vortices. ${ }^{1,47}$ In addition, Bourgeois et al. ${ }^{3,45}$ $\left(A R=4, R e=1.2 \times 10^{4}\right)$ and Hosseini et al..$^{47}(A R=8$, Re $=1.2 \times 10^{4}$ ) proposed two closely related overall models for the 3D vortex structure, defined as "Bourgeois's Model" here, in which two looped or half-looped principle cores are alternatively shed from either side of a square cylinder and are connected at both ends through the connector strands. The Bourgeois's model is a fully connected entity in the whole wake and is relatively more complicated than the hairpin vortex model put forward by Dousset and Pothérat, ${ }^{19}$ the Wang's model $^{1}$ (which is constituted with two basic components, viz., the symmetrically or anti-symmetrically arranged spanwise vortex rolls.), and several disconnected 3D vortex models (in which tip vortices, base vortices, and spanwise vortices are separate flow structures, as presented by Kawamura et al. ${ }^{4}$ Sumner, ${ }^{43}$ Pattenden et al. ${ }^{33}$ and Krajnovic ${ }^{5}$ ). The diversity of types of vortex-shedding structures around a finite cylinder is not totally unexpected due to the complexity of the flow and the large number of influencing parameters (e.g., $A R, R e$, and $\delta / D)$.

The purpose of this study is to analyze the influence of the $R e$ number on the time-averaged streamlines and pressure contours, on the type of the mean streamwise vortex structure and on the instantaneous spanwise vortex-shedding model in the scenario of a finite square cylinder. Six relatively low $R e$ numbers (i.e., 50, 100, 150, 250, 500, and 1000) at a fixed $A R$ (i.e., 4) are considered in an effort to reduce the dimensions of the influencing parameters.

\section{CONFIGURATION AND NUMERICAL MODEL}

\section{A. Test configuration}

A similar geometric configuration as that used by Saha ${ }^{16}$ is employed in the present study. As illustrated in Fig. 1, a 


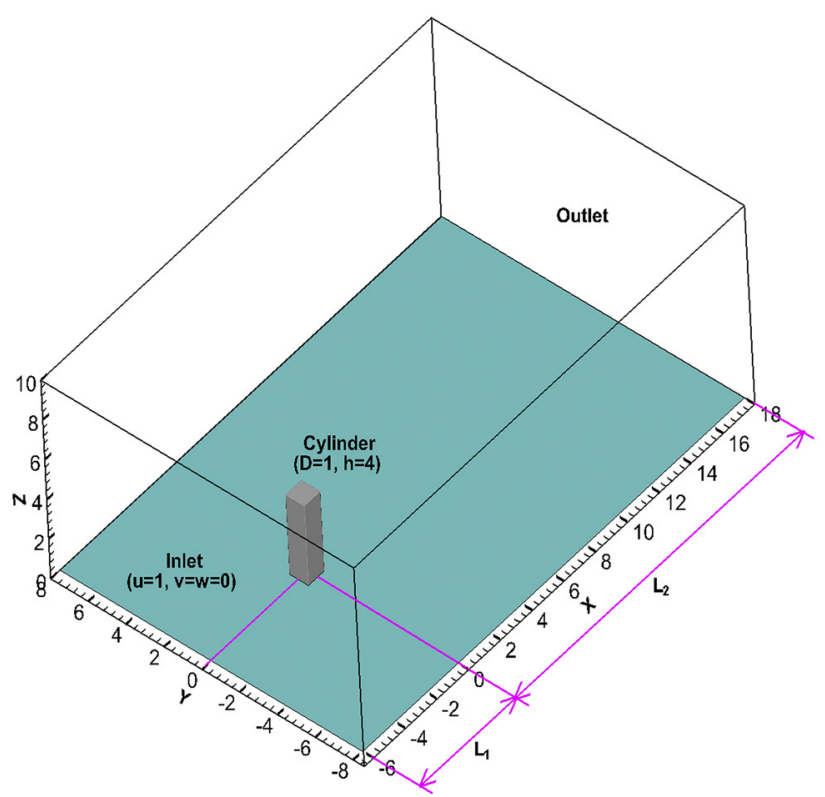

(a) The Computational Domain

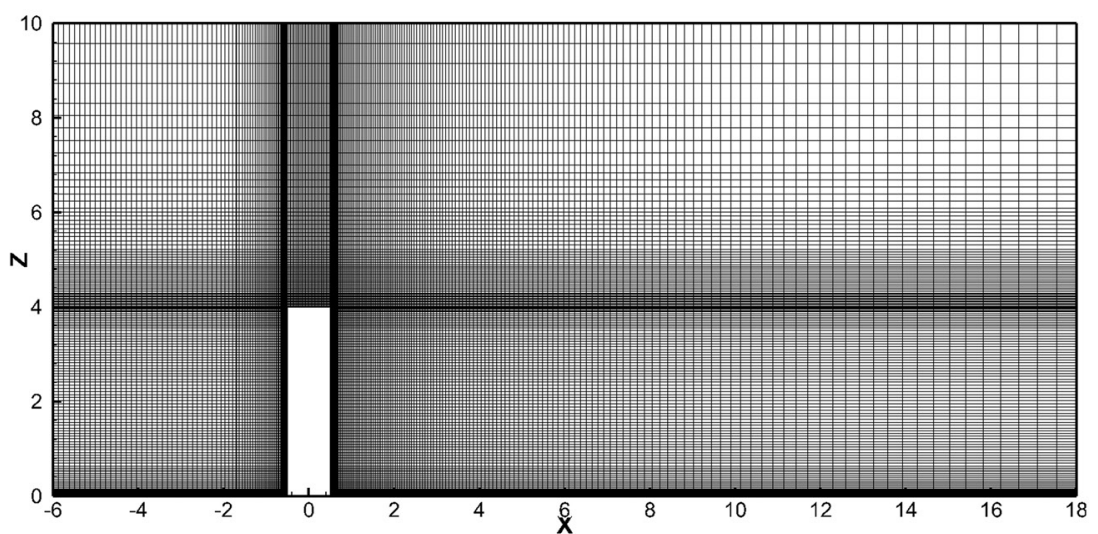

(b) The Level-3 Mesh on the Symmetry (Y=0) Plane

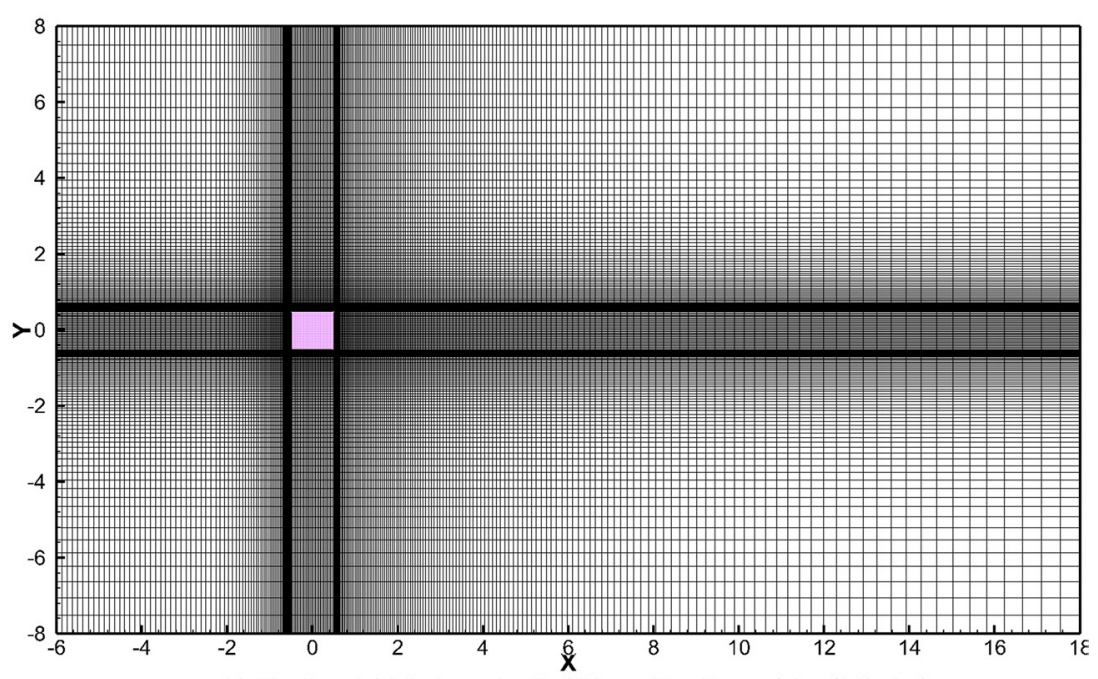

(c) The Level-3 Mesh on the $Z=4$ Plane (Top Face of the Cylinder)
FIG. 1. Schematic of the computational domain and the level-3 mesh. finite square cylinder with a non-dimensional width of $D=1$ and a non-dimensional height of $h=4$ is vertically mounted on a plane boundary, and the (streamwise) length, (transverse) width, and (spanwise) height of the computational domain are, respectively, $L=24, W=16$, and $H=10$ (which gives an area blockage ratio of $2.5 \%$ ). Further, the junction section between the cylinder and the bottom wall is centered at the origin of the coordinate system, which means that the inlet boundary is located at $6 D$ upstream of the cylinder and the outlet boundary is situated at $18 D$ downstream of the cylinder (i.e., $L_{1}=6 D$, $L_{2}=18 D$ ). It has been verified by $\mathrm{Saha}^{16}$ that the spanwise domain size, which is equal to the sum of the cylinder height 
and six times that of the cylinder diameter (i.e., $H=h+6 D$ ), is reasonable because even a significant increase of this size will only lead to a percentage difference of about $0.2 \%$ in terms of the drag coefficient and the dominant vortex-shedding frequency.

\section{B. Numerical model}

The non-dimensional continuity equation and 3D incompressible Navier-Stokes (N-S) equations employed in this study can be described as follows: ${ }^{48-50}$

$$
\begin{aligned}
\frac{\partial u_{i}}{\partial x_{i}} & =0, \\
\frac{\partial u_{i}}{\partial t}+\nabla \cdot\left(u_{i} u_{j}\right) & =-\nabla p+\frac{1}{R e} \nabla^{2} u_{i},
\end{aligned}
$$

where the subscripts $i$ and $j$ indicate the $i$ th and $j$ th components of the Cartesian coordinate, respectively, $u$ and $p$ represent the non-dimensional velocity and pressure, respectively, and $t$ is the non-dimensional time. The numerical results of six Reynolds numbers will be presented in this study, namely, Re $=50,100,150,250,500$, and 1000. Four kinds of boundary conditions are involved as follows:

1. Inlet: For the velocity field, a fixed uniform velocity is prescribed (i.e., $u_{I}=1, v_{I}=w_{I}=0$ ), and, for the pressure field, the zero-gradient condition is imposed (i.e., $\partial p_{I} / \partial n$ $=0$ ).

2. Outlet: For the velocity field, the convective outflow boundary condition, as described by Krajnovic, ${ }^{5}$ Saha, ${ }^{16}$ and Hwang and Yang, ${ }^{18}$ has been adopted,

$$
\frac{\partial \phi}{\partial t}+\vec{u}_{c} \frac{\partial \phi}{\partial n}=0
$$

where $\phi$ denotes all the three velocity components (viz., $u, v$, and $w$ ) and $\vec{u}_{c}$ is the advective velocity at the outlet boundary. For the pressure field, the homogeneous Dirichlet condition is utilized at the outlet boundary, namely, $p=0$.

3. The bottom wall and the surface of the obstacle: No-slip impermeable boundary condition is prescribed for the velocity field (i.e., $u=v=w=0$ ), and the zero-gradient condition is employed for the pressure field.

4. Top and lateral boundaries of the computational domain: Free-slip condition is prescribed, which means that the velocity component normal to the boundary is zero (i.e., $u_{b \perp}=0$ ) and the normal gradients of both the pressure and the velocity component tangential to the boundary are zero (i.e., $\partial u_{b / /} / \partial n=\partial p_{b} / \partial n=0$, where $u_{b / /}$ is the tangential velocity component).

The numerical simulations were carried out by using the Open Source Field Operation and Manipulation (OpenFOAM) $\mathrm{C}++$ libraries. Specifically, icoFoam, one of the standard solvers provided by OpenFOAM, is selected to solve the nondimensional incompressible Navier-Stokes (N-S) equations. The Pressure Implicit with Splitting of Operators (PISO) ${ }^{50}$ algorithm is employed to deal with the pressure-velocity coupling on a collocated grid system in the context of the finite volume method (FVM). The Gauss linear scheme is chosen to discretize the convection term, the diffusion term, and the pressure gradient term in the momentum equation, and the
Euler implicit scheme is adopted for the temporal discretization. By employing the aforementioned numerical schemes, the final form of the non-dimensional momentum equations for an orthogonal mesh can be expressed by the following formula:

$$
\begin{aligned}
\left(\frac{\vec{u}^{t+\Delta t}-\vec{u}^{t}}{\Delta t}\right)+\frac{1}{V_{c}} \sum_{f=1}^{6} F_{f} \vec{u}_{f}^{t+\Delta t}= & -\frac{1}{V_{c}} \sum_{f=1}^{6} \vec{A}_{f} p_{f}+\frac{1}{R e} \cdot \frac{1}{V_{c}} \\
& \times \sum_{f=1}^{6} \vec{A}_{f} \cdot\left(\nabla \times \vec{u}^{t+\Delta t}\right)_{f},
\end{aligned}
$$

where $\Delta t$ is the adopted time step, $c$ is the center of the considered control volume, $V_{c}$ is the volume of the control cell, $f=1$, $2,3,4,5$, and 6 is the centroid of the cell face, $\vec{A}_{f}$ is the area vector of the cell face, and $F_{f}=\vec{A}_{f} \cdot \vec{u}_{f}^{t}$ is the volumetric flux at the cell face. Especially, the linear interpolation scheme is utilized to acquire the values of $\vec{u}_{f}^{t+\Delta t}, p_{f}$, and $\left(\nabla \times \vec{u}^{t+\Delta t}\right)_{f}$ in Eq. (4). For further details of these numerical schemes, readers can refer to the references corresponding to temporal and spatial discretization schemes. ${ }^{49,51,52}$

\section{VALIDATION AND GRID CONVERGENCE}

The present numerical model was validated against the results reported by Saha, ${ }^{16}$ where a case identical to one of the test cases in this study (viz., $R e=250, A R=4$ ) was presented. For the other five test cases (i.e., $R e=50,100,150,500$, and 1000), no published numerical or experimental results are available; therefore, the grid-dependency study is performed in order to guarantee sufficient grid resolution. As displayed in Table I, four levels of non-uniform structured meshes, with $13.96,8.24,6.18$, and $3.98 \times 10^{6}$ grid points, respectively, are employed to check the grid-independency, and the number of grid points, $N_{x} \times N_{y} \times N_{z}$, distributed on each side of the cylinder varies with different grid configurations. Since the accuracy of the boundary-layer simulation has an influence on the flow around a finite cylinder, the distance between the first grid point and the bottom wall is also reported in Table I (defined as near-wall grid size). Consequently, the boundary-layer thickness at the location of the cylinder (in the absence of the cylinder) is $\delta / D \approx 1.16$ at $R e=50, \delta / D$ $\approx 0.86$ at $R e=100, \delta / D \approx 0.72$ at $R e=150, \delta / D \approx 0.58$ at $\operatorname{Re}=250, \delta / D \approx 0.43$ at $R e=500$, and $\delta / D \approx 0.31$ at $\operatorname{Re}$ $=1000$. Besides, to improve both the temporal accuracy and the numerical stability, different time steps are adopted for different grid resolutions to ensure that the maximum CourantFriedricks-Lewey (CFL) number in all the cases is smaller than 0.25 .

Table I also provides the computed mean drag coefficients $C_{d}$ and the Strouhal numbers $S_{t}$ for all the seven grid levels (at $R e=250$ ), which are defined as

$$
C_{d}=F_{x} /\left(\rho U^{2} D h / 2\right), \quad S_{t}=f_{s} D / U,
$$

where $U$ is the free stream velocity, $F_{x}$ is the drag force in the $x$ direction, obtained by integrating the pressure and shear stress over all cylinder surfaces, and $f_{s}$ is the dominant frequency of the transverse fluctuating velocity at the location $(x, y, z)=(16.0,0.0,2.0)$ in the far-wake. Overall, at 
TABLE I. Validation and grid convergence for a square cylinder of $A R=4$.

\begin{tabular}{|c|c|c|c|c|c|c|c|}
\hline $\begin{array}{l}\text { Mesh } \\
\text { name }\end{array}$ & $\begin{array}{l}\text { Grid } \\
\text { resolution }^{\mathrm{a}}\end{array}$ & $\begin{array}{c}\text { Number of } \\
\text { nodes }\left(\times 10^{6}\right)\end{array}$ & $\begin{array}{l}N_{x} \times N_{y} \\
\quad \times N_{z}^{\mathrm{b}}\end{array}$ & $\begin{array}{l}\text { Near-wall } \\
\text { grid size }\end{array}$ & $\begin{array}{c}\text { Time } \\
\text { step }(\Delta t)\end{array}$ & $\begin{array}{c}\text { Strouhal } \\
\text { number }(\operatorname{Re}=250)^{\mathrm{c}}\end{array}$ & $\begin{array}{c}\text { Mean drag } \\
\text { coefficient }(\operatorname{Re}=250)\end{array}$ \\
\hline Level-1 & $215 \times 148 \times 125$ & 3.98 & $32 \times 32 \times 77$ & 0.007 & 0.002 & 0.121 & 1.186 \\
\hline Level-2 & $244 \times 171 \times 148$ & 6.18 & $39 \times 39 \times 93$ & 0.004 & 0.001 & 0.123 & 1.208 \\
\hline Level- $3^{\mathrm{d}}$ & $262 \times 185 \times 170$ & 8.24 & $42 \times 42 \times 104$ & 0.001 & 0.0008 & 0.123 & 1.213 \\
\hline Level- $4^{\mathrm{e}}$ & $303 \times 228 \times 202$ & 13.96 & $48 \times 48 \times 118$ & 0.0006 & 0.0005 & $\cdots$ & $\ldots$ \\
\hline Saha's coarse level ${ }^{16}$ & $152 \times 112 \times 98$ & 1.66 & $26 \times 26 \times 71$ & 0.025 & 0.002 & 0.120 & 1.19 \\
\hline Saha's middle level ${ }^{16}$ & $177 \times 136 \times 120$ & 2.89 & $34 \times 34 \times 83$ & 0.01 & 0.002 & 0.124 & 1.23 \\
\hline Saha's fine level ${ }^{16}$ & $204 \times 150 \times 132$ & 4.04 & $42 \times 42 \times 92$ & 0.005 & 0.002 & 0.125 & 1.23 \\
\hline
\end{tabular}

${ }^{\mathrm{a}}$ In these structured meshes, the grid points are clustered near the bottom wall and the cylinder surfaces (Figs. 1(b) and 1(c)).

${ }^{\mathrm{b}} N_{x}, N_{y}$, and $N_{z}$, respectively, represent the number of grid points distributed on each side of the cylinder.

${ }^{\mathrm{c}}$ The Strouhal number $\left(S_{t}\right)$ is calculated at the location $(x / D=16, y / D=0$, and $z / D=2)$ for the transverse fluctuating velocity.

${ }^{\mathrm{d}}$ Level-3 is the adopted grid of case-1 $(R e=50)$, case-2 $(R e=100)$, case-3 $(\operatorname{Re}=150)$, case-4 $(\operatorname{Re}=250)$, and case-5 $(\operatorname{Re}=500)$.

${ }^{\mathrm{e}}$ Level-4 is the employed grid of case-6 (viz., $\left.R e=1000\right)$ in this study in order to enhance the accuracy as much as possible.

$R e=250$, level-2 and level-3 almost result in the same mean drag coefficient and Strouhal number (the corresponding percentage errors are, respectively, $0.42 \%$ and $0.00 \%$.), which are slightly different from that obtained by Saha, ${ }^{16}$ as shown in Table I. However, this deviation is deemed as reasonable in consideration of the large difference in mesh resolution. It should be emphasized that, although the level-3 mesh is fine enough to get the grid-independent solution for $R e \leq 500$ (viz., from case-1 to case-5), when it comes to $R e=1000$ (viz., case-6), the level-4 mesh is necessary in order to achieve the grid-convergence, as displayed in the Appendix. In order to reach the fully developed flow regime in the wake, all the simulations were first run over approximate 42 flowthrough periods (viz., $T_{0} \approx 42 \times\left(L_{1}+L_{2}\right) / U \approx 1000$ ). Then, the flow fields were averaged for another non-dimensional time interval of 1000 to yield statistically independent results.

Table II summarizes the variation of the mean drag coefficients $C_{d}$, the Strouhal number $S_{t}$, the type of the mean streamwise vortices, and the instantaneous spanwise vortexshedding model with the increase of Re. It is obvious that the value of $C_{d}$ first decreases, then increases during this process, and reaches the minimum around $R e=250$. Besides, the critical Reynolds number between steady and unsteady flows in this study lies in the range of $100 \leq R e_{c r i}<150$, which is consistent with the results of Dousset and Potherat ${ }^{19}$ ( $A R$ $=4$, square cylinder; $R e_{c r i} \approx 150$, large $\delta$ value). This value is relatively larger than its counterpart in the $2 \mathrm{D}$ scenario in view of that $R e_{c r i-2 D}=30-60$ (Breuer et al. $\left.{ }^{10}\right), \operatorname{Re}_{c r i-2 D}<70$ $\left(\right.$ Okajima $\left.^{53}\right)$, and $R e_{c r i-2 D}=54\left(\right.$ Klekar and Patankar $\left.{ }^{54}\right)$. The value of $S_{t}$ falls in $S_{t}=[0.100,0.123]$, being in accordance with the numerical results of Wang and Zhou ${ }^{1}(A R=7$, $R e=9300$, and $S t=0.110)$, Saeedi et al. ${ }^{12}(A R=4, R e$ $=12000$, and $S t=0.106)$, and Bourgeois et al. ${ }^{3,45}(A R=4$, $R e=12000$, and $S t=0.100 \pm 0.003)$. When it comes to the types of mean streamwise vortices and the spanwise vortexshedding model (serving as the two main innovations of this paper), various kinds of vortex structures exist in the range of the Re considered in this study, which will be detailed in Sec. IV.

\section{RESULTS AND DISCUSSION}

\section{A. Mean velocity and pressure fields}

In this section, the mean velocity and pressure fields are investigated by examining the time-averaged streamlines and pressure contours in several characteristic planes.

\section{Two characteristic planes}

Figs. 2 and 3 show the time-averaged streamlines and pressure contours on two characteristic sections, namely, the symmetry plane $(Y=0)$ and the mid-height plane $(Z$ $=2$ ). As expected, a higher $R e$ number leads to a stronger negative pressure behind the cylinder. The dashed purple line depicts the separation line originating from the leading

TABLE II. Variation of the mean drag coefficient, Strouhal number, and vortex structure with the Re number.

\begin{tabular}{lcccc}
\hline \hline$R e$ & $\begin{array}{c}\text { Mean drag } \\
\text { coefficient }\end{array}$ & $\begin{array}{c}\text { Strouhal } \\
\text { number }\end{array}$ & $\begin{array}{c}\text { Mean streamwise vortex } \\
\text { structure }\end{array}$ & $\begin{array}{c}\text { Spanwise vortex-shedding } \\
\text { model }\end{array}$ \\
\hline 50 & 1.675 & $\ldots$ & "Quadrupole Type" & Steady flow \\
100 & 1.314 & $\ldots$ & "Six-Vortices Type" & "C-Reverse-C-Hairpin Vortex Model" \\
150 & 1.222 & 0.116 & & $\begin{array}{c}\text { "Hymmetric Shedding) } \\
250\end{array}$ \\
500 & 1.213 & 0.123 & "Dipole Type" & "C-Reverse-C-Hairpin Vortex Model" \\
1000 & 1.298 & 0.113 & 0.106 & Symmetric/Anti-Symmetric Shedding) \\
\hline \hline
\end{tabular}

${ }^{a}$ In the literature, $S t=0.110$ for Wang and $\mathrm{Zhou}^{1}(A R=7, R e=9300), S t=0.106$ for Saeedi et al. ${ }^{12}(A R=4, R e=12000)$, and $S t=0.100 \pm 0.003$ for Bourgeois et al. ${ }^{3,45}(A R=4, R e=12000)$. 

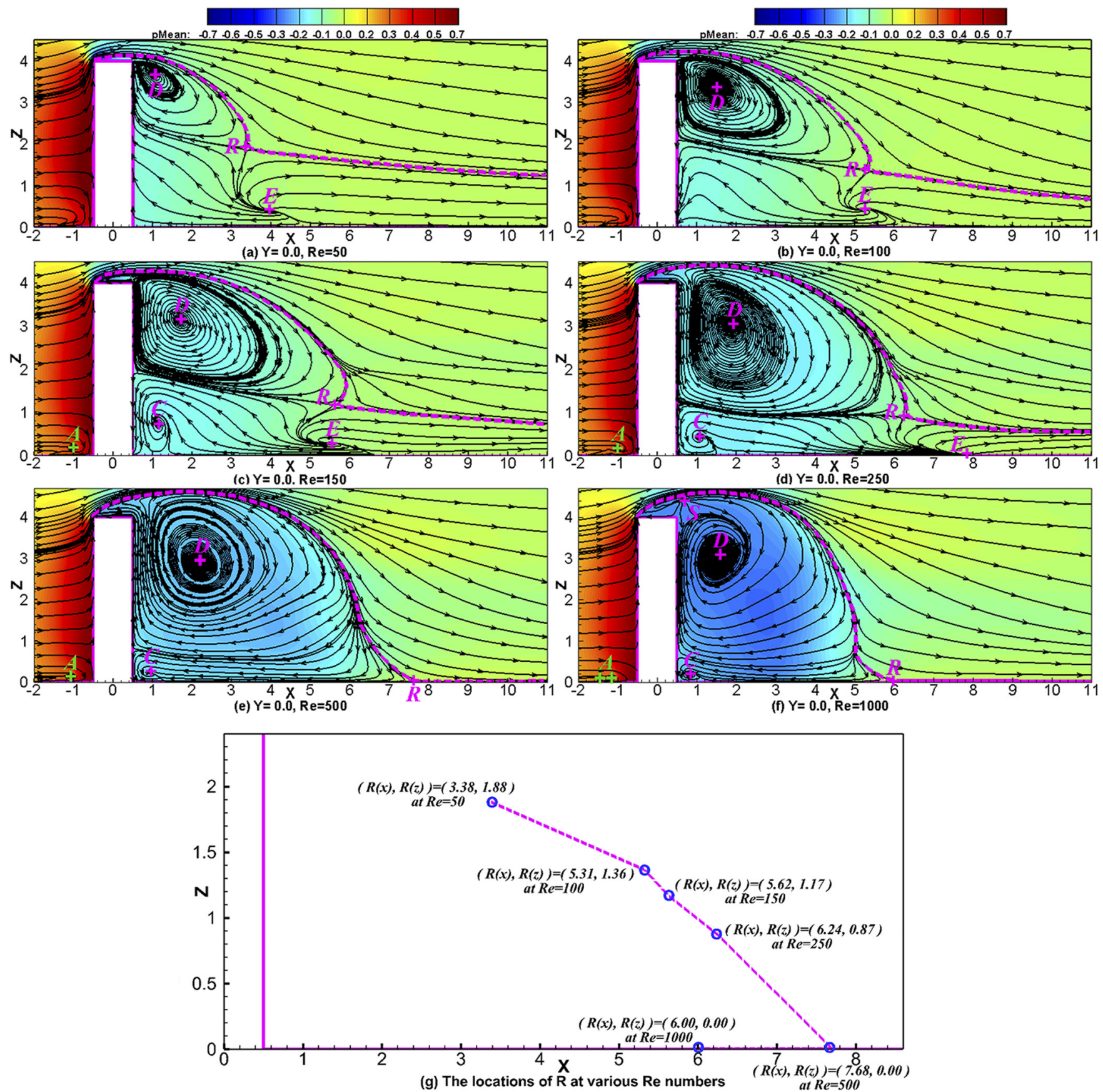

FIG. 2. Time-averaged streamlines and pressure fields on the symmetry plane $(Y=0)$ at different $R e$ numbers: (a) $R e=50$; (b) $R e=100$; (c) $R e=150$; (d) $R e$ $=250$; (e) $R e=500$; (f) $R e=1000$; (g) the locations of $R$ at various $R e$ numbers.

corner of the cylinder, which is the dividing line between the outer zone and the influence region of the backflow. Actually, the separating line in the symmetry plane directly reflects the strength of the downward flow from the tip (i.e., downwash ${ }^{1,2,21}$ ) and plays an important role in the formation of flow structures.

As shown in Fig. 2, in contrast to case-5 (i.e., $R e=500$ ) and case-6 (i.e., $R e=1000$ ), the other four cases have a different streamline pattern in the mid-transverse plane because of the relatively weak downwash and the relatively thick boundarylayer, which can be deduced from the fact that the separation line in Figs. 2(a)-2(d) fails to reach the bottom wall. This results in the impingement point (denoted by the capital letter
$R$ in Fig. 2) occurring above the base. Consequently, an obvious upward flow (defined as the upwash by Wang et al. ${ }^{25}$ and Bourgeois et al. ${ }^{3}$ ), which comes from the source point $E$ and clashes with the downwash along the $X$ direction, can be observed in Figs. 2(a)-2(d), and this upwash flow has a significant influence on several aspects of the flow field, such as the mean streamwise vortices, which will be described later on in detail. Besides, Fig. 2(g) presents the locations of the impingement point $R$ for all the six Re numbers. Moreover, Fig. 3 compares the time-averaged streamlines on the mid-span plane for each of the cases. It is clear that, except for case-2 $(R e=100)$, the remaining five cases share the same streamline profile, including two symmetrically distributed spiral nodes (i.e., $A$ and $B$ in 


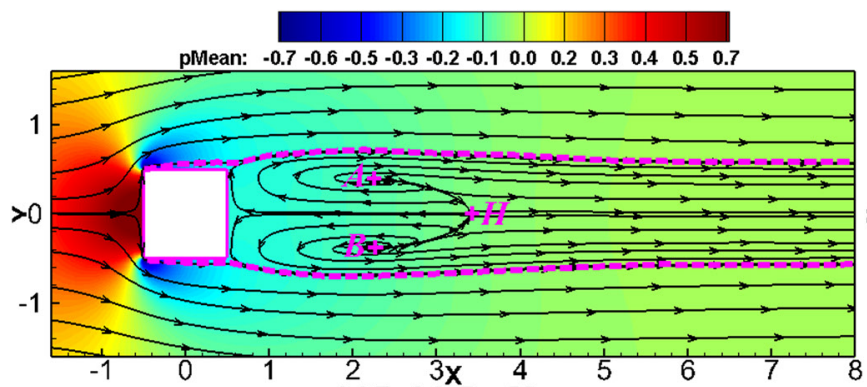

(a) $Z=2.0, \operatorname{Re}=50$

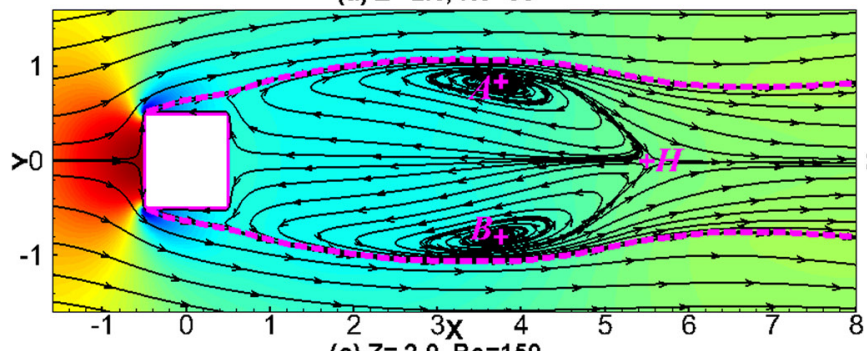

(c) $Z=2.0, \mathrm{Re}=150$

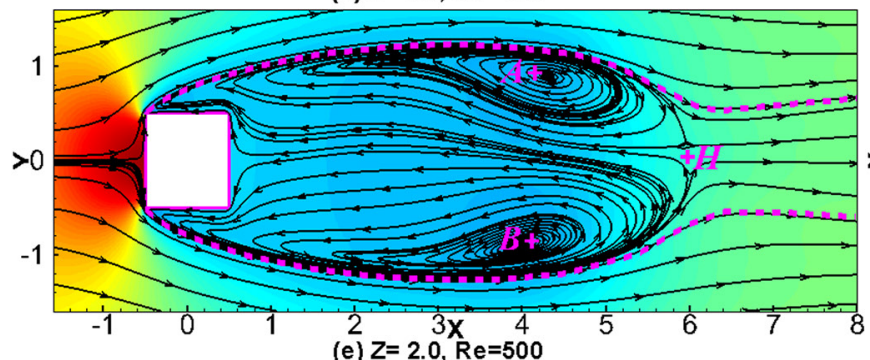

(e) $Z=2.0, \mathrm{Re}=500$

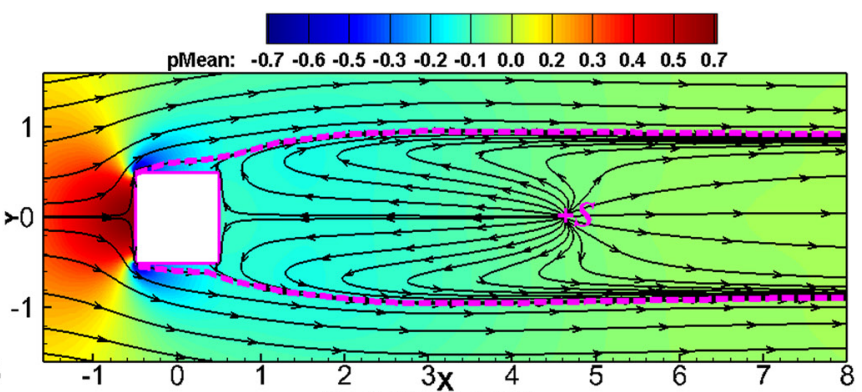

(b) $Z=2.0, \mathrm{Re}=100$

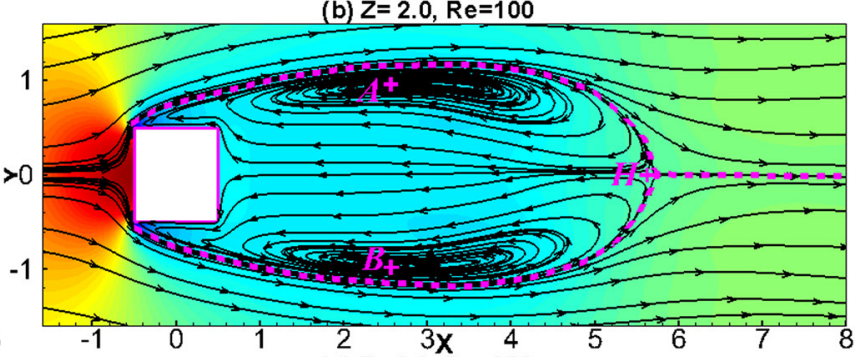

(d) $Z=2.0, \mathrm{Re}=250$

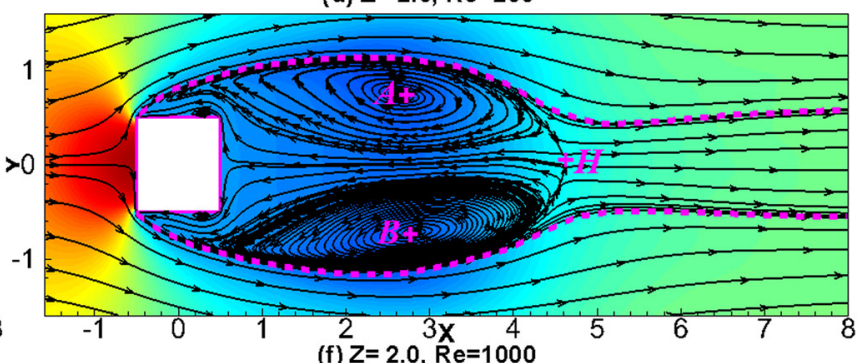

(f) $Z=2.0, R_{e}=1000$

FIG. 3. Time-averaged streamlines and pressure fields on the mid-span plane $(Z=2)$ at different $R e$ numbers: (a) $R e=50$; (b) $R e=100$; (c) $R e=150$; (d) $R e$ $=250 ;$ (e) $R e=500 ;$ (f) $R e=1000$.

Figs. 3(a) and 3(c)-3(f)) and one saddle node (i.e., $H$ in Figs. 3(a) and 3(c)-3(f)).

Especially, Figs. 2(a)-2(d) and 2(g) demonstrate that, when $R e \leq 250$, the spanwise size of the upwash flow near the base will diminish with the increase of $R e$ (considering that $R(z)=1.88$ in Fig. 2(a), $R(z)=1.36$ in Fig. 2(b), $R(z)$ $=1.17$ in Fig. 2(c), and $R(z)=0.87$ in Fig. 2(d), where $R(z)$ is the $Z$-coordinate value of the impingement point $R$.), but the streamwise range of the upwash flow will monotonically increase during this process (considering that $R(x)=3.38$ in Fig. 2(a), $R(x)=5.31$ in Fig. 2(b), $R(x)=5.62$ in Fig. 2(c), and $R(x)=6.24$ in Fig. 2(d), where $R(x)$ is the $X$-coordinate value of the impingement point $R$ ). However, when $R e \geq 500$, the $X$-position of the impingement point $R$ will decrease with the increase of the Re number, and, consequently, the reattachment point at $R e=500$ (viz., $R(x)=7.68$ in Fig. 2(e)) is farther from the cylinder than its counterpart at $R e=1000$ (viz., $R(x)$ $=6.00$ in Fig. 2(f)). Overall, on the symmetry plane, the size of the downwash recirculation region (its center is denoted by $D$ in Fig. 2) will first increase then decrease with the growing of the Reynolds number and reaches the maximum around $R e$ $=500$ (Fig. 2(e)). On the contrary, the vertical dimension of the upwash recirculation region (its possible center is indicated by $C$ in Fig. 2) will monotonically decrease with the increase of $R e$.

Additionally, at $R e=50$ and $R e=100$, the flow is steady and no spiral node $A$ (representing the time-averaged horseshoe vortex) can be observed in front of the cylinder, but, at $R e=150,250$, and 500, there is one horseshoe-vortex spiral point on the symmetry plane, marked by $A$ in Figs. 2(c)-2(e), and the number of the horseshoe vortices increases up to 2 at $R e=1000$ (Fig. 2(f)).

\section{Flow patterns on the cylinder surfaces}

Fig. 4 provides the topology of the mean flow in planes at a non-dimensional distance of 0.0001 from the cylinder surfaces (i.e., $d_{n}=0.0001$ ) for all the six Reynolds numbers. On the top surface, no backflow region (no separation) can be observed at $R e=50$, but, with the increase of $R e$, the recirculation region (separated flow) appears, whose area becomes larger and larger. As shown in Figs. 4(b)-4(f), two spiral-flow centers (i.e., $A$ and $B$ ) are symmetrically distributed within the backflow region, and their locations shift away from the $Y=0$ plane as $R e$ increases, namely, $Y_{A \& B}= \pm 0.277$, $\pm 0.407, \pm 0.455, \pm 0.496$, and \pm 0.541 at the five $R e$ values with an increasing sequence. It should be noted that $A$ and $B$ are actually located outside the top surface at $R e=1000$, as confirmed by the coordinate value. There is a separation line connected between the points $A$ and $B$ near the front edge of the top surface, and this separation line moves towards the front edge with the increase of $R e$ in the range of $R e=100-1000$. Fig. 4(f) also reveals that at $R e=1000$ a source node $S$ exists near the trailing edge of the tip, which is actually originated from the source point $S$ in the symmetry plane (as shown in Fig. 2(f)). However, at $R e=150,250$, and 500, the backflow above the top surface in the $Y=0$ plane is initiated from the 


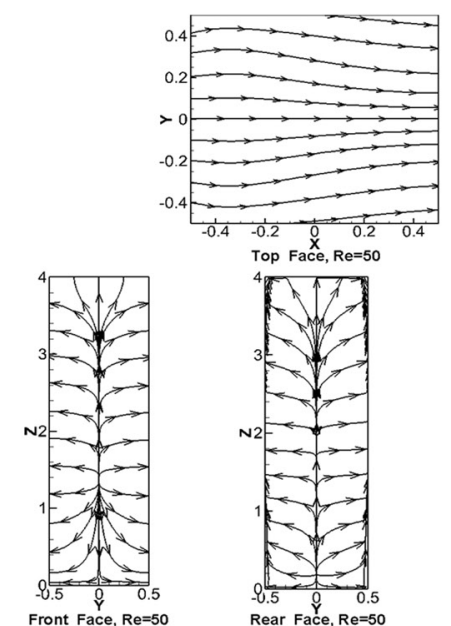

(a)
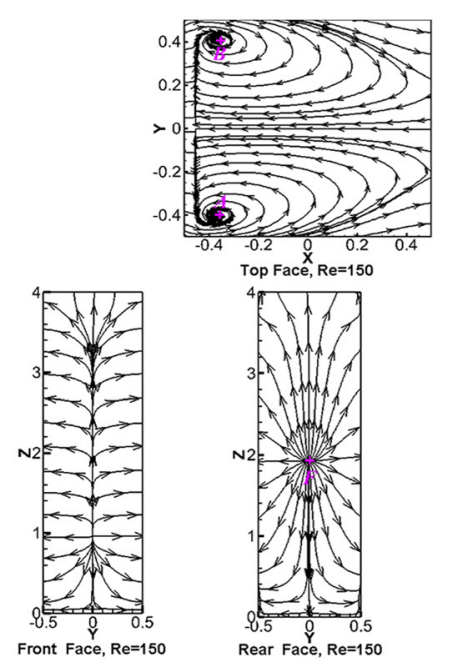

(c)

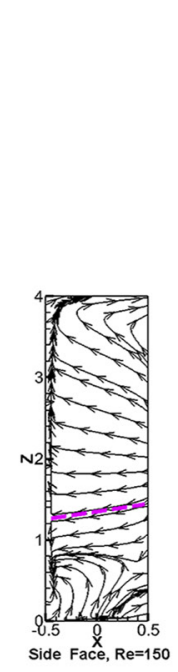

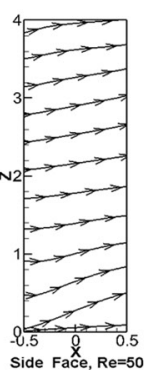
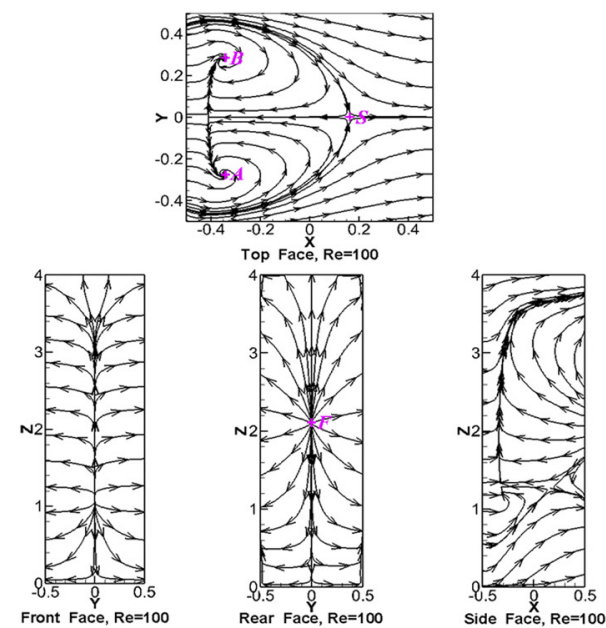

(b)
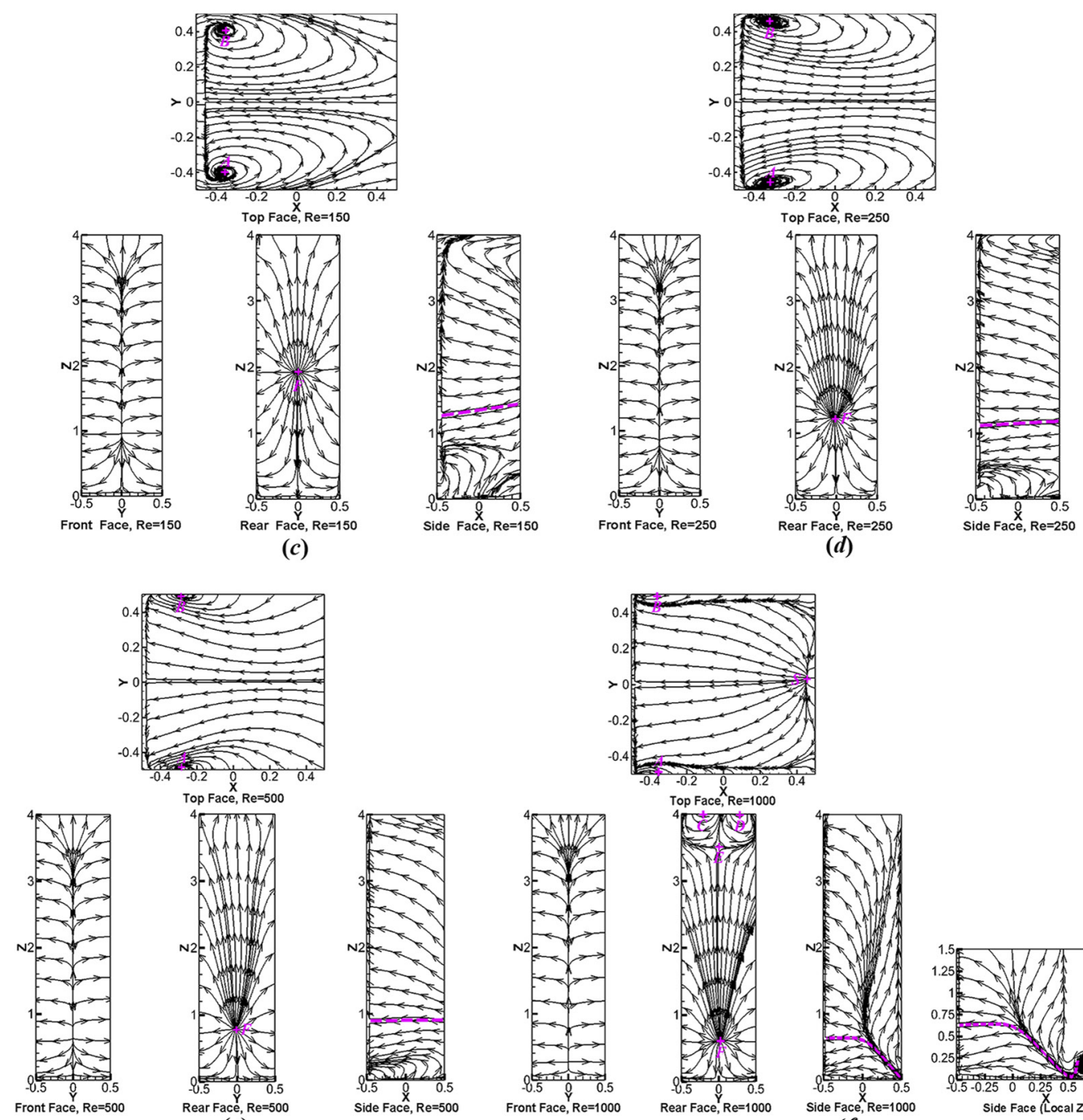

(e)
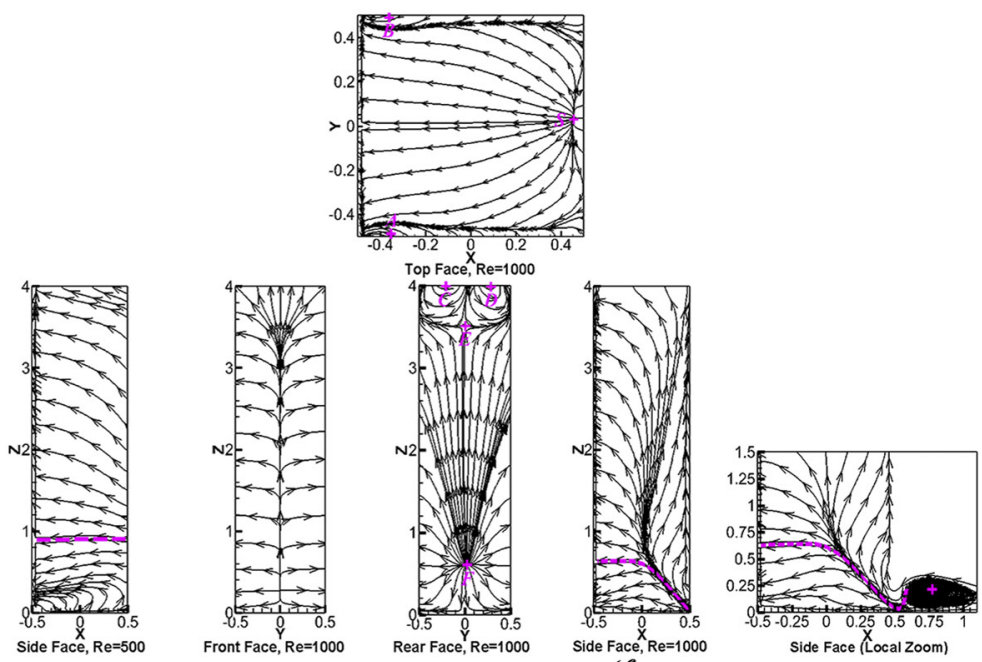

(f)

FIG. 4. Time-averaged streamlines on cylinder surfaces. (a) $\operatorname{Re}=50$; (b) $\operatorname{Re}=100$; (c) $\operatorname{Re}=150$; (d) $\operatorname{Re}=250$; (e) $\operatorname{Re}=500$; (f) $\operatorname{Re}=1000$.

downwash recirculation region behind the cylinder (i.e., $D$ in Fig. 2(c)-2(e)).

On the front face, a vertical separation line lies in the center along the spanwise direction, and the streamlines along most of the span show the two-dimensional feature (at least in the neighbor-hood of the obstacle). However, near the two ends of the cylinder, the two-dimensionality of the streamlines vanishes because of the effect of the tip and the boundary layer. Furthermore, with the increase of $R e$, the thickness of the boundary layer becomes smaller, and consequently, the 
two-dimensional flow region on the front face will extend toward the bottom wall, as shown in Fig. 4.

On the rear face, except for $R e=50$ (viz., Fig. 4(a)), the flow comes from a source point, denoted by $F$ in Figs. 4(b)-4(f). With the increase of $R e$, the height of the source point will continuously decrease (namely, $Z_{F}=2.10,1.92$, $1.23,0.78$, and 0.60 for the five $R e$ values with an increasing sequence), and the curvature of the mean streamlines penetrating through the top edge will increase. Consequently, a pair of counter-rotating spiral flows is formed near the tip at $R e=1000$, expressed by $C$ and $D$ in. Fig. 4(f). Actually, the two spiral-flow centers are slightly higher than the top edge (i.e., $Z_{C \& D} \approx 4.11$ ), but they are still marked out in the figure. In addition, the point $F$ is situated at the center line of the rear face in the present study, which is different from an asymmetric location reported in Ref. 16. The present result (in terms of the location of the point $F$ ) appears to be reasonable, considering the time-averaged property and the geometrical symmetry of the computational domain.

As shown in Figs. 4(c)-4(f), for all the four unsteady cases, on the lateral face of the cylinder, a (partly) quasihorizontal dividing line (i.e., the dashed purple line) can be identified, above/below which the mean streamlines turn to the upside/downside. Especially, at $R e=1000$, the partly quasihorizontal dividing line, passing through the lower right corner (i.e., $X=0.5, Z=0$ ), essentially stems from the spiral center on the lateral plane, being similar to the point $C$ in Fig. 2(f).

\section{B. Mean streamwise vortices}

The appearance of mean streamwise vortices is one of the most remarkable properties of the finite-cylinder wake, and previous studies showed that either a dipole type or a quadrupole type of the mean streamwise vortex structure can be observed in the wake depending on the aspect ratio with a critical value of $A R_{c}=3-5$, as presented by Wang and Zhou, ${ }^{1}$ Sumner and Heseltine, ${ }^{2}$ Bourgeois et al.,${ }^{3,45}$ Sumner et al. ${ }^{21}$ Porteous et al.,${ }^{27}$ Pattenden et al.,${ }^{33}$ and Frederich et $a l^{44}$ Although the influence of $A R$ on the mean streamwise vortex structure has been extensively discussed, ${ }^{1-3,21}$ a systematic study on the effect of the Re number on the types of the mean streamwise vortices is absent in the existing literature, which actually serves as the main content of this section.

The $\lambda_{2}$ criterion, put forward by Jeong and Hussain, ${ }^{55}$ is selected to display the mean streamwise vortex structure in the wake because this criterion not only captures the pressure minimum in a plane perpendicular to the vortex axis at high $R e$ numbers but also accurately defines vortex cores at low $R e$ numbers, ${ }^{3,45,55}$

$$
\begin{aligned}
S_{i j} & =\frac{1}{2}\left(U_{i, j}+U_{j, i}\right), \Omega_{i j}=\frac{1}{2}\left(U_{i, j}-U_{j, i}\right), \\
M_{i j} & =S^{2}+\Omega^{2}=S_{i k} S_{k j}+\Omega_{i k} \Omega_{k j}, \\
\lambda_{2} & =\text { the second eigenvalue of } M_{i j},
\end{aligned}
$$

where the subscript comma denotes partial differentiation and the summation convention is used for the indices $i, j$, and
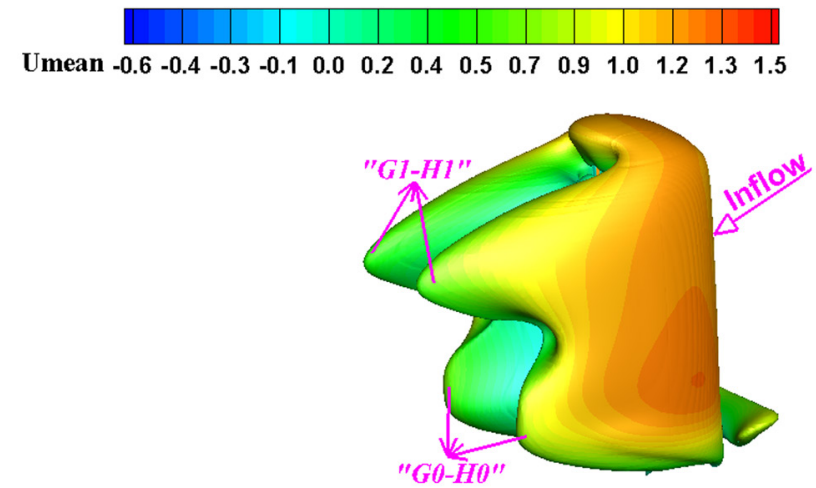

(a) Mean Streamwise Vortex Structure in the Wake, $\operatorname{Re}=50, \lambda_{2}=-0.024$

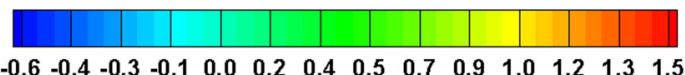

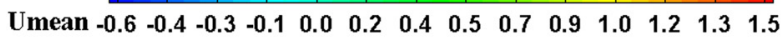

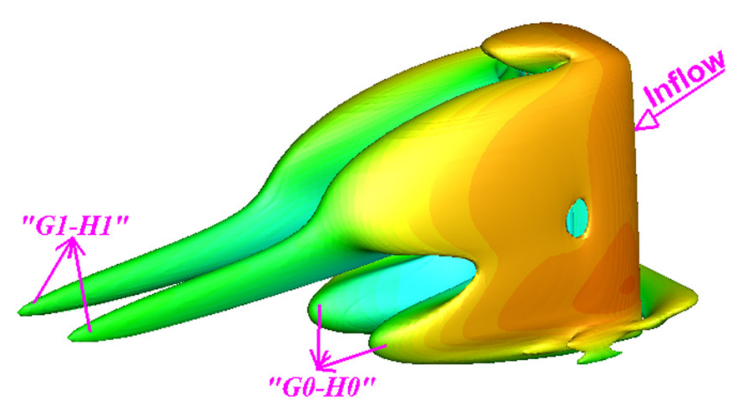

(b) Mean Streamwise Vortex Structure in the Wake, $\operatorname{Re}=100, \lambda_{2}=-0.024$

FIG. 5. "Quadrupole Type" mean streamwise vortex structure in the wake at $R e=50\left(\lambda_{2}=-0.024\right)$ and $R e=100\left(\lambda_{2}=-0.024\right)$ coloured by the magnitude of the mean velocity in the $X$-direction (i.e., umean).

$k$ (which, respectively, indicate the three components of the Cartesian coordinates). $S_{i j}$ and $\Omega_{i j}$ are the symmetric and antisymmetric parts of the velocity gradient tensor $\nabla U_{i j}$. If the second eigenvalue of the $M_{i j}$ tensor is locally negative (viz., $\lambda_{2}<0$ ), a vortex core can then be detected at this location.

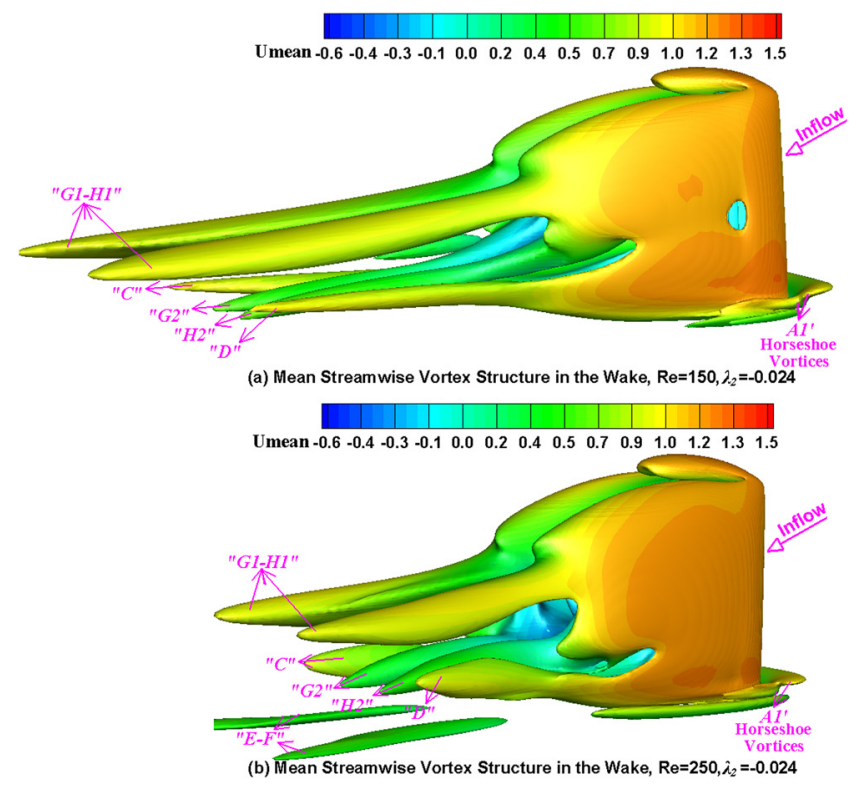

FIG. 6. "Six-Vortices Type" mean streamwise vortex structure in the wake at $\operatorname{Re}=150\left(\lambda_{2}=-0.024\right)$ and $\operatorname{Re}=250\left(\lambda_{2}=-0.024\right)$ coloured by the magnitude of umean. 


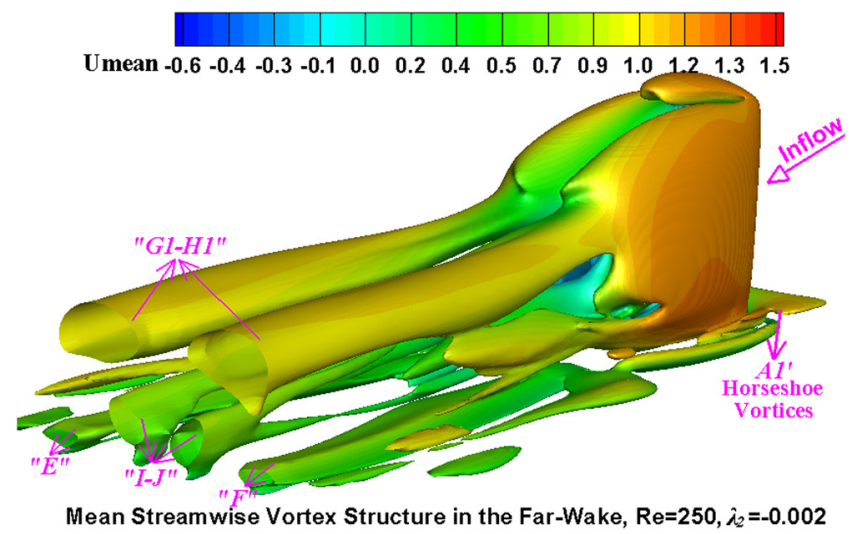

FIG. 7. Mean streamwise vortex structure will be transformed from "SixVortices Type" into "Quadrupole Type" at $R e=250\left(\lambda_{2}=-0.002\right)$ in the far-wake region.

Figs. 5-8 show the 3D vortex cores of the mean flow field identified by utilizing the $\lambda_{2}$ criterion for all the six cases. It should be emphasized that, at least two distinct conclusions can be drawn from these figures, which are different from the previous observations and actually serve as one of the main innovation points of this study, as discussed in the following paragraphs.

First, with the increase of $R e$, three types of mean streamwise vortex structures are identified, namely, the "Quadrupole Type" at $R e=50$ and $R e=100$, the "Six-Vortices Type" at Re $=150$ and $R e=250$, and the "Dipole Type" at $R e=500$ and $R e=1000$. As illustrated in Fig. 5, the "Quadrupole Type" is comprised of mean streamwise base vortices (i.e., "G0-H0," caused by the upwash flow) and mean streamwise tip vortices (i.e., "G1-H1," caused by the downwash flow). Fig. 6 shows that the "Six-Vortices Type" is constituted by "G1-H1," descending mean tip vortices (i.e., "G2-H2"), and one pair of counter-rotating vortices caused by the bending of the mean streamlines induced by the upward flow (i.e., " $C$ - $D$ ”). In fact, Fig. 6(b) suggests that two pairs of counter-rotating vortices (i.e., " $C-D$ " and " $E-F$ ") are generated from the bending of the mean streamlines near the base caused by the upward flow at $R e=250$. Considering that the extent and strength of " $E-F$ " are relatively small when compared with that of " $C-D$," " $E$ $F$ " vortices are not deemed as one part of the main structures of mean streamwise vortices. As demonstrated in Fig. 8, the "Dipole Type" only consists of one pair of mean streamwise tip vortices "G1-H1". As far as we know, only the "Dipole Type" and the "Quadrupole Type" have been presented in the existing literature by varying the $A R$ value; therefore, it is the first time that the "Six-Vortices Type" mean streamwise vortices are reported, which can be treated as the transitional structure between the other two types. Special attention should be paid to Figs. 6(b) and 7, which indicate that the mean streamwise vortex structure transforms from "Six-Vortices Type" in the middle-wake region into "Quadrupole Type" in the far-wake region at $R e=250$, where " $G 2-H 2$ " and " $C-D$ " vanish and the extent of " $E-F$ " is relatively small when compared with that of "G1-H1" and mean streamwise base vortices-farwake (i.e., "I-J"). The transition process between the "Six-Vortices Type" in the middle-wake (i.e., Fig. 6(b)) and the "Quadrupole Type" in the far-wake (i.e., Fig. 7) will be detailed later on for the purpose of ascertaining the formation mechanism of the newly proposed "Six-Vortices Type" mean streamwise vortices.

Second, serving as an extension of the conclusions drawn from previous research, it is put forward that the type of the mean streamwise vortices in the wake is determined by whether or not the downward flow from the cylinder tip can reach the bottom wall, namely, whether or not the downwash in the upper part and the upwash in the lower part coexist in the symmetry plane, depending on the combined influence of $R e, A R$, and $\delta / D$. For example, Figs. 2(e), 2(f),
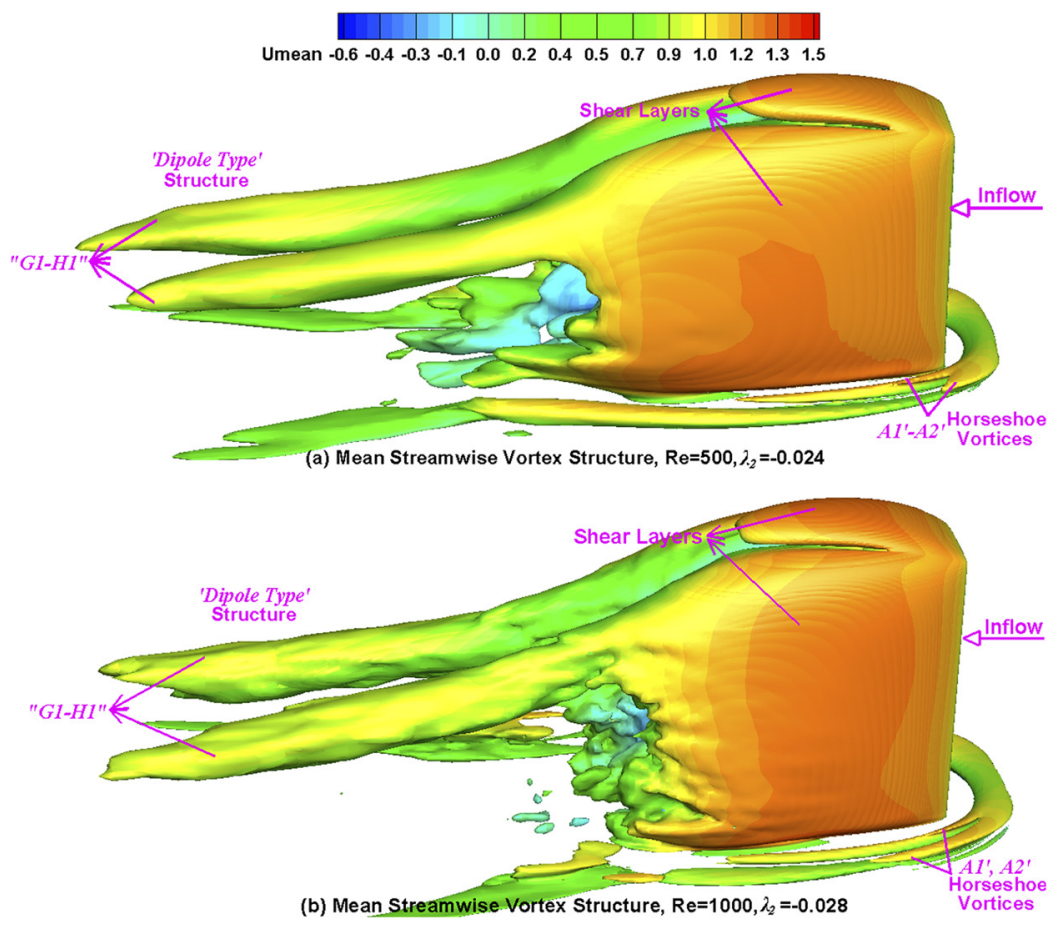

FIG. 8. "Dipole Type" mean streamwise vortex structure in the wake at $R e=500\left(\lambda_{2}=-0.024\right)$ and $R e=1000\left(\lambda_{2}\right.$ $=-0.028)$ coloured by the magnitude of umean. 

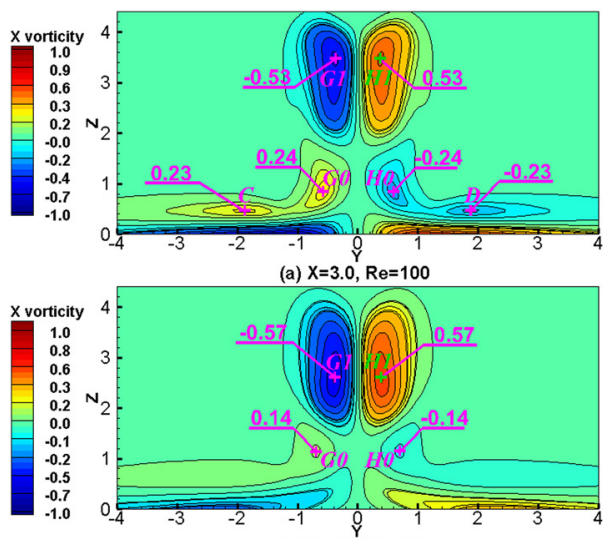

(c) $X=5.0, R e=100$

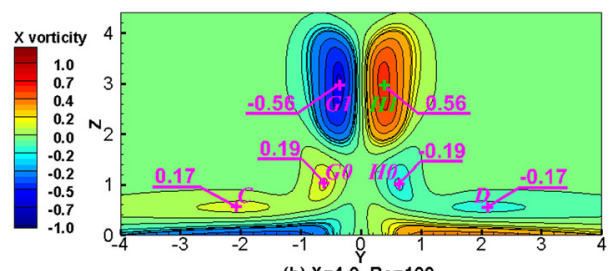

(b) $X=4.0, R e=100$
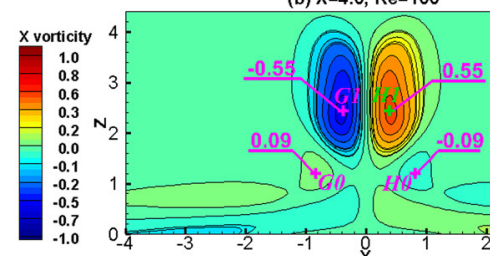

$0.09((+))((0))))_{-0.00}$

(1) -0.09

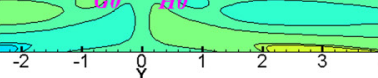

(d) $X=6.0, R e=100$

FIG. 9. The "Quadrupole Type" evolution of the mean streamwise vortices between the near and far wakes at $R e$ =100. (a) $X=3.0$; (b) $X=4.0$; (c) $X$ $=5.0 ;$ (d) $X=6.0$. and 8 indicate that, when the downwash can reach the base (viz., the impingement point $R$ lies on the bottom wall in the symmetry plane), only a pair of counter-rotating mean streamwise tip vortices (viz., "Dipole Type") is observed in the wake at $R e=500$ and $R e=1000$, but Figs. 2(a)-2(d), 5, and 6 show that, when the downwash fails to reach the bottom wall (viz., the impingement point $R$ occurs above the base in the symmetry plane), either "Quadrupole Type" or "Six-Vortices Type" mean vortex structure is formed in the wake, in spite of the same $A R$ value for all the six cases. In other words, Figs. 5-8 clearly illustrate that, even for a fixed $A R$ value, all the three types of profiles are possible. However, in the existing literature, ${ }^{1-3,21,33,45}$ it was believed that the mean streamwise vortex pattern is determined by the $A R$ value, namely, the dipole type when $A R \leq A R_{c}$ and the quadrupole type when $A R>A R_{c}$. We underline the fact that, on one hand, for a prescribed $R e$ (with the same $\delta / D$ value), the $A R$ value does play a critical role on the relative strength of the downwash, and this leads to previous findings that the type of mean streamwise vortices in the wake is dependent on the $A R$ value. On the other hand, for a given $A R$ value, the occurrence of the upwash flow (being the root cause of the mean streamwise base vortices) near the base hinges on both $R e$ and $\delta / D$. The results shown in Figs. 2(a), 2(b), and 5 suggest that the combination of low $R e$ and large $\delta / D$ values is conductive to the coexistence of the downwash and upwash in the symmetry plane, namely, the formation of the quadrupole type.
Considering that the transition process from the complex streamwise vortex system in the near-wake to the simple streamwise vortex pattern in the far-wake has not been satisfactorily explained until now, ${ }^{3,20}$ the remaining part of this section will focus on the evolution of mean streamwise vortices between the near and far wakes with the aid of mean streamlines and mean vorticity contours. In view of the definition of vorticity (viz., $\Omega=\nabla \times U$, namely, the strength and profile of the vorticity field is determined by the velocity gradient), the vortex cores identified by the mean streamlines may not be completely consistent with that observed from the mean vorticity contours. As confirmed in Figs. 6 and 23 in Ref. 1, Fig. 3 in Ref. 2, Figs. 7 and 8 in Ref. 21, Figs. 12 and 13 in Ref. 33, and Figs. 9-16 in this article, the locations of the vortex cores identified by the mean streamlines sometimes deviate from those obtained by the vorticity contours to a certain degree. It is possible that, some vortex cores are visible by means of the vorticity contours, but no corresponding spiral flow can be observed by the streamlines and vice versa. Therefore, it is necessary to combine the mean streamlines with the mean vorticity contours in order to effectively explain the evolution of mean streamwise vortices.

Figs. 9 and 10 present the quadrupole-type evolution of mean streamwise vortices in the wake for case-2 (i.e., Re $=100)$, being in accordance with the results of Fig. 5(b). Actually, case-1 (i.e., $R e=50$ ) shares almost the same transition process, which is omitted here for simplicity. It
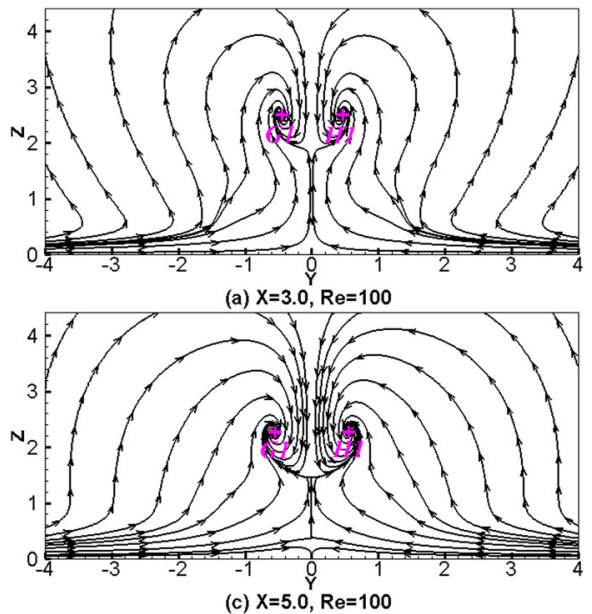

(c) $X=5.0, R e=100$
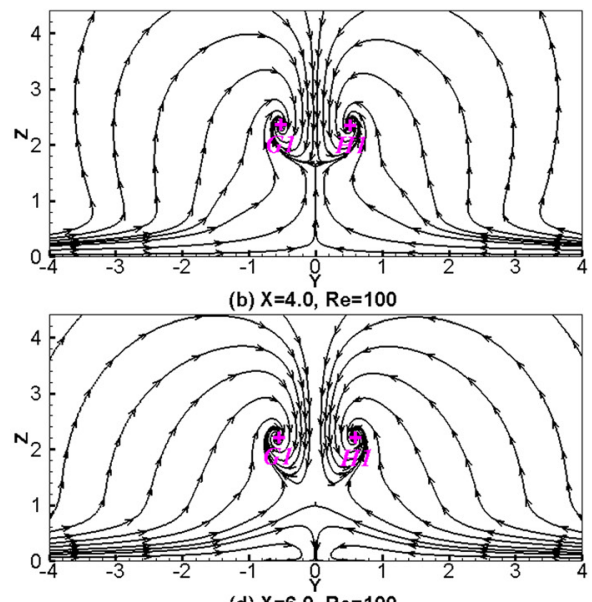

(d) $X=6.0, R_{e}=100$
FIG. 10. The evolution of the timeaveraged streamlines between the near and far wakes at $R e=100$. (a) $X=3.0$; (b) $X=4.0$; (c) $X=5.0$; (d) $X=6.0$. 

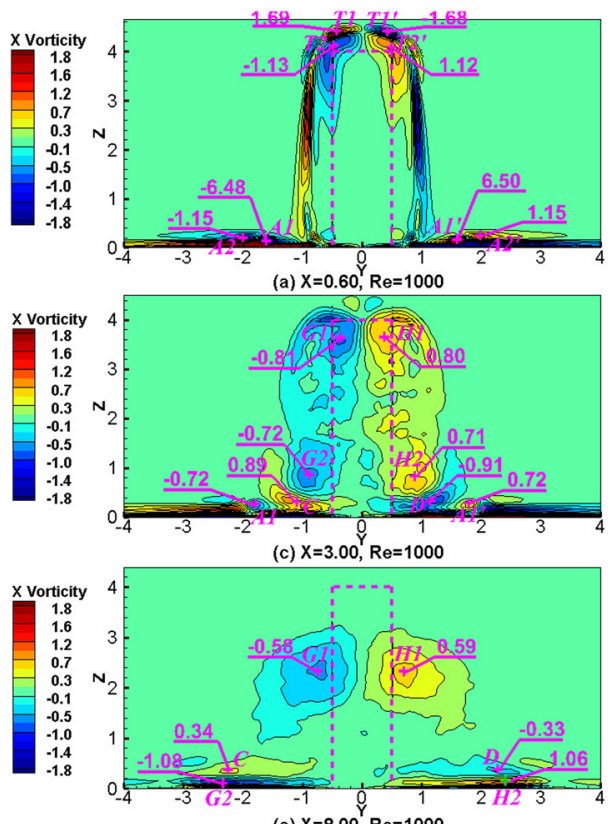

(e) $X=8.00, R e=1000$
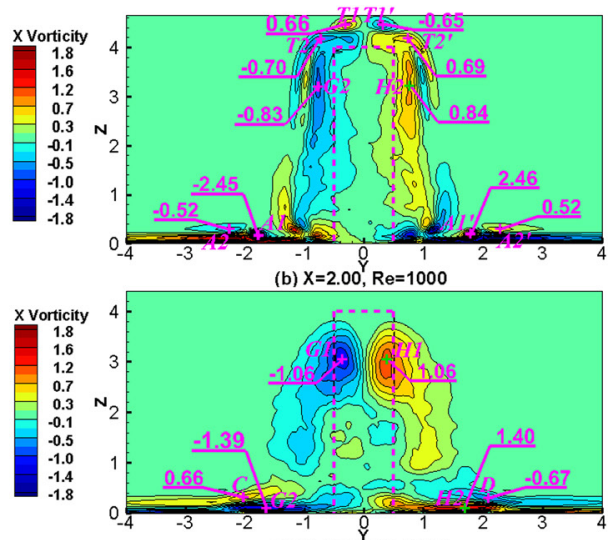

(d) $X=5.00, R_{e}=1000$

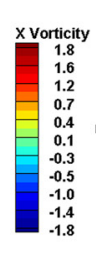

Vorticity
1.8
1.6
1.2
0.7
0.4
0.1
0.1
-0.3
-0.5
-1.0
-1.4
-1.8

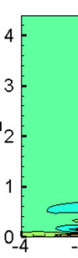

FIG. 11. The "Dipole Type" evolution of the mean streamwise vortices between the near and far wakes at $R e$ =1000. (a) $X=0.60$; (b) $X=2.00$; (c) $X$ $=3.00$; (d) $X=5.00$; (e) $X=8.00$; (f) $X$ $=10.0$. is obvious that, with the increasing distance from the cylinder, the strength of G1-H1 (mean streamwise tip vortices, caused by the downwash flow from the cylinder tip) first increases then decreases (reaching the maximum value around $X=5$ (i.e., $\left|\Omega_{x}\right|=0.57$ )), but the strength of $\mathrm{G0}-\mathrm{HO}$ (mean streamwise base vortices, caused by the upwash flow near the base) monotonically decreases during this process. Other conclusions include that the height of $G 1-H 1$ continuously decreases when moving along the downstream direction, and two pairs of mean streamwise vortices (viz., G0-H0 and $C-D$ ) are formed due to the bending of the mean streamlines caused by the upward flow from the base at the $X=3.0$ section.
Figs. 11 and 12 depict the dipole-type evolution of mean streamwise vortices in the wake for $\operatorname{Re}=1000$, being in accordance with the results of Fig. 8(b). In fact, $R e=500$ shares almost the same transition process, which is omitted here for simplicity. At least three evolution stages can be identified in the wake for the dipole-type evolution. Stage-I refers to the front part of the region between the rear surface of the cylinder and the center of the downwash recirculation zone in the symmetry plane (i.e., the point $D$ in Figs. 2(e) and 2(f)), such as $X=0.60$ at $R e=1000$. As displayed by Figs. 11 and 12(a), the upward flow dominates the streamwise planes situated within this region, and two pairs of counter-rotating timeaveraged tip vortices are formed near the free-end, namely,

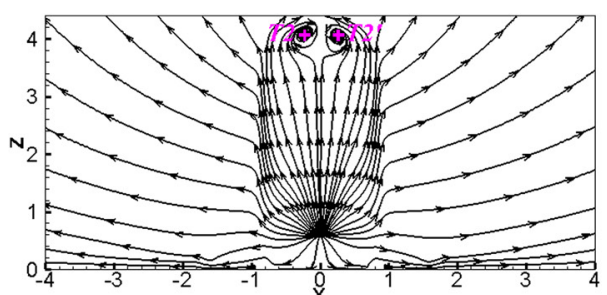

(a) $X=0.60, R e=1000$

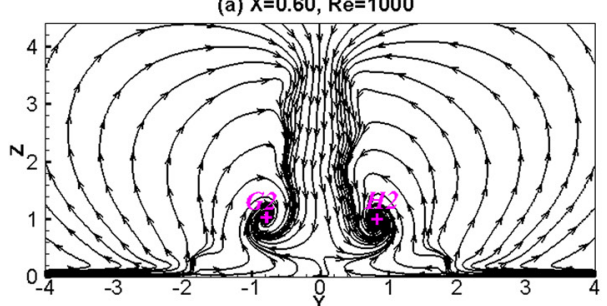

(c) $\mathrm{X}=3.00, \mathrm{Re}=1000$

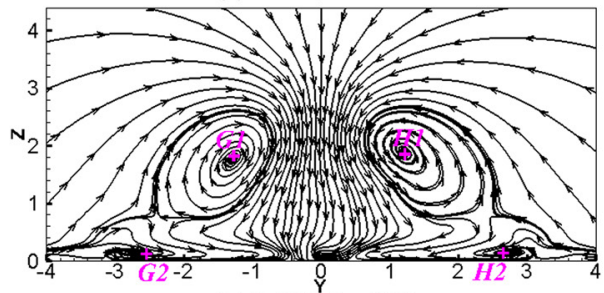

(e) $X=8.00, R e=1000$

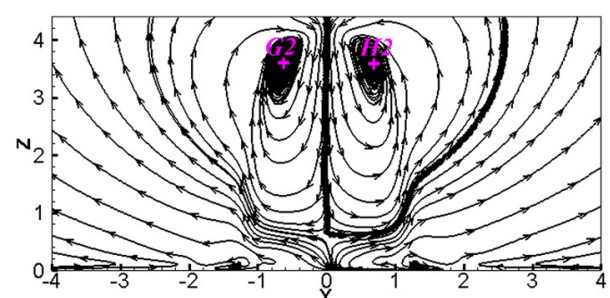

(b) $\mathrm{X}=2.00, \mathrm{Re}=1000$

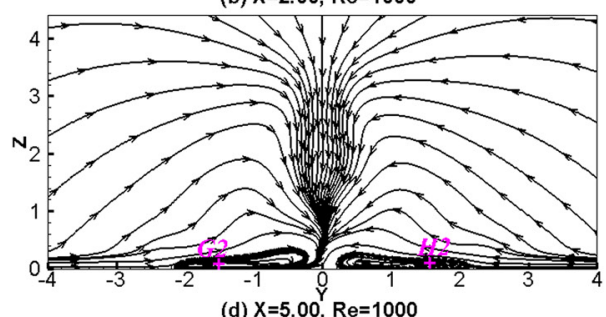

(d) $X=5.00, R e=1000$

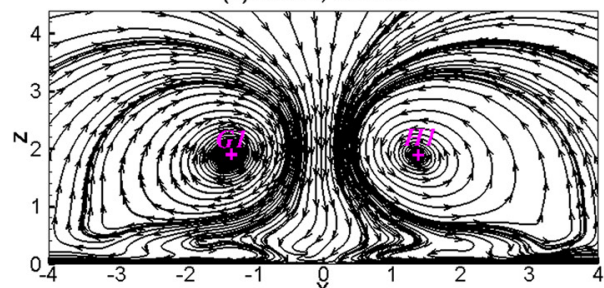

(f) $X=10.0, R e=1000$
FIG. 12. The evolution of the timeaveraged streamlines between the near and far wakes at $R e=1000$. (a) $X=0.60$; (b) $X=2.00$; (c) $X=3.00$; (d) $X=5.00$; (e) $X=8.00$; (f) $X=10.0$. 

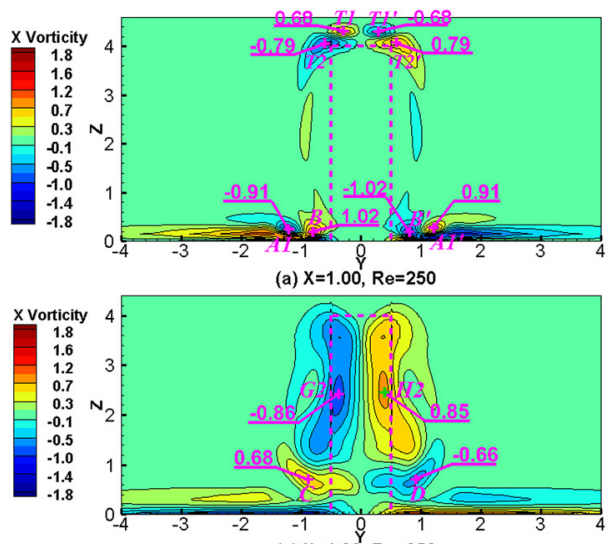

(c) $X=4.00, R e=250$

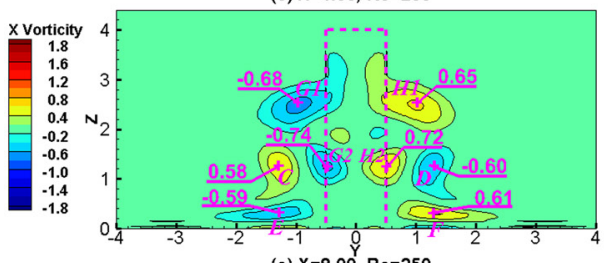

(e) $X=8.00, \operatorname{Re}=250$
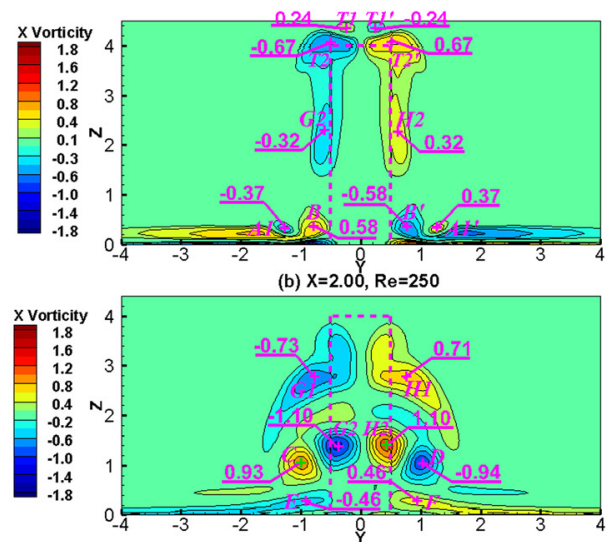

(d) $X=6.00, R e=250$

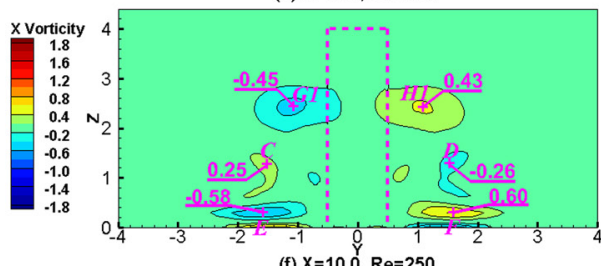

FIG. 13. The "Six-Vortices Type" evolution of the mean streamwise vortices between the near and far wakes at $R e$ = 250: Part-1. (a) $X=1.00$; (b) $X=2.00$; (c) $X=4.00$; (d) $X=6.00$; (e) $X=8.00$; (f) $X=10.0$ tornado-like tip vortices $T 1-T 1^{\prime}$ and trailing side tip vortices $T 2-T 2^{\prime}$. Similar to the conclusions reached in Ref. 38, $T 1-T 1^{\prime}$ evolves from two centers of the spiral flow on the top surface, namely, $A$ and $B$ in Fig. 4(f), which can be verified by showing the vorticity contours in the plane traversing $A$ and $B$ (but omitted here for simplicity). Besides, $T 2-T 2^{\prime}$ originates from two leading corners of the tip; however, for circular cylinders, the corresponding vortex pair is actually produced by the periphery of the free-end. ${ }^{38}$ During the stage-I, the strength of $T 1-T 1^{\prime}, T 2-T 2^{\prime}$, and two pairs of horseshoe vortices ( $A 1-A 1^{\prime}$ and $\left.A 2-A 2^{\prime}\right)$ continuously decreases with the increase of distance from the obstacle. Stage-II is characterized by the emergence of the descending mean tip vortices $\mathrm{G} 2-\mathrm{H} 2$ and the coexistence of $G 1-H 1$ and $G 2-H 2$, such as $X=2-8$ at $R e$ $=1000$ (viz., Figs. 11(b)-11(e)). When the downwash is formed in the upper part, $\mathrm{G} 2-\mathrm{H} 2$ is observed, as shown in Figs. 11 and 12(b). When moving further downstream, the strength of the downwash in the upper region of the streamwise plane increases continuously, and consequently the mean streamwise tip vortices $\mathrm{G} 1-H 1$ are formed in the vorticity contours figure, but no corresponding spiral flow is observed from the mean streamline representation, as confirmed by Figs. 11, 12(c), and 12(d). At further downstream locations, when the strength of the downwash in the upper zone is large enough, a pair of spiral flow corresponding to $G 1-H 1$ eventually appears in the mean streamline figure, as presented in Fig. 12(e). As for G2-H2,

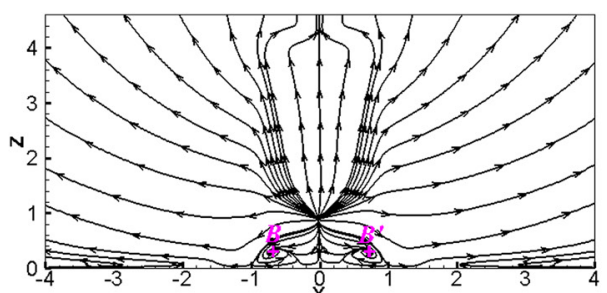

(a) $X=1.00, R e=250$

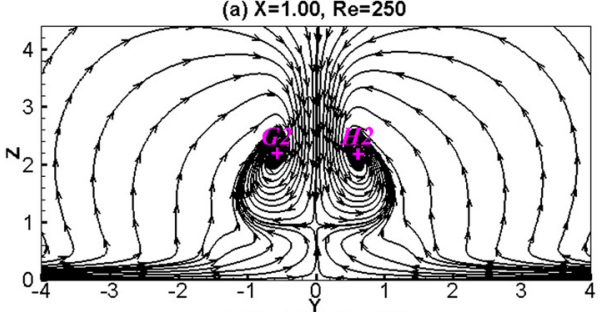

(c) $X=4.00, R e=250$

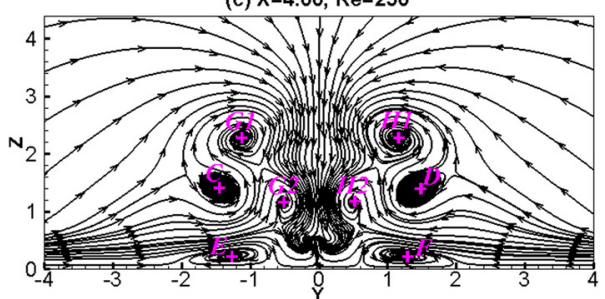

(e) $X=8.00, \operatorname{Re}=250$

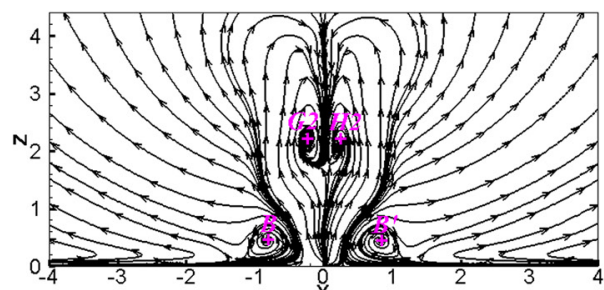

(b) $\mathrm{X}=2.00, \mathrm{Re}=250$

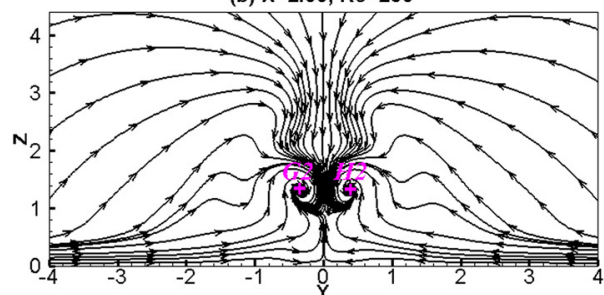

(d) $X=6.00, R e=250$

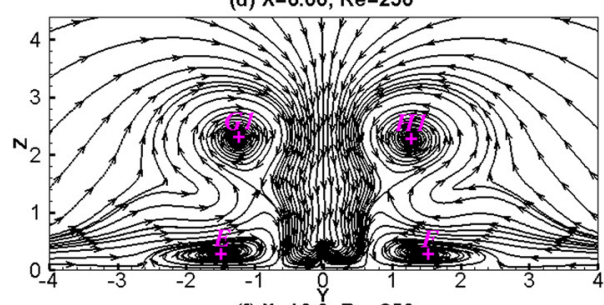

(f) $X=10.0, R e=250$
FIG. 14. The evolution of the timeaveraged streamlines between the near and far wakes at $R e=250$ : Part-1. (a) $X$ $=1.00$; (b) $X=2.00$; (c) $X=4.00$; (d) $X$ $=6.00 ;$ (e) $X=8.00$; (f) $X=10.0$. 


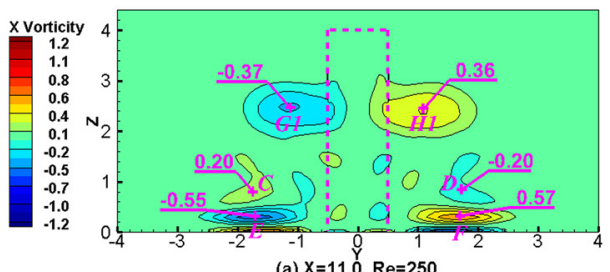

(a) $X=11.0, R e=250$

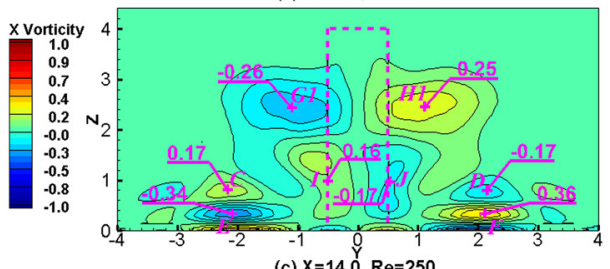

(c) $X=14.0, R e=250$

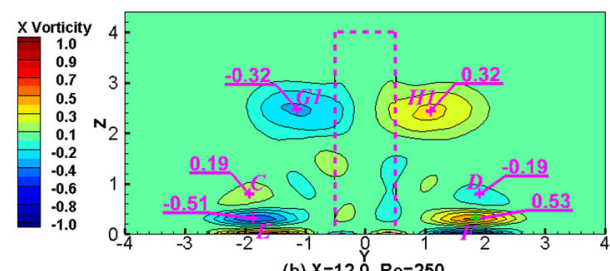

(b) $X=12.0, R_{e}=250$

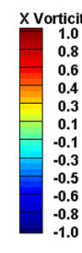

Vorticity
1.0
0.8
0.6
0.4
0.4
0.3
0.1
-0.1
-0.3
-0.5
-0.6
-0.8
-1.0

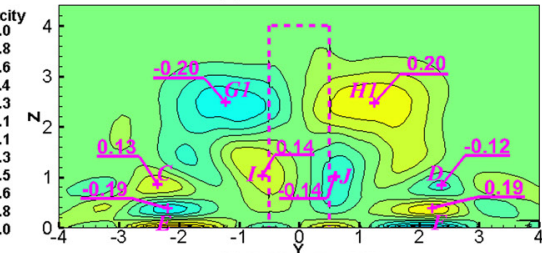

(d) $X=16.0, R e=250$
FIG. 15. The "Six-Vortices Type" evolution of the mean streamwise vortices between the near and far wakes at $R e$ =250: Part-2. (a) $X=11.0$; (b) $X=12.0$; (c) $X=14.0$; (d) $X=16.0$ it is found that this pair of vortices, being originated from the downward flow in the upper region, will be transported downwards with the increase of the $X$ value till the height of the impingement point $R$. After that, $G 2-H 2$ will move laterally away from the centreline, its strength continuously decreases, and finally it disappears in both of the streamline and vorticity representations. Additionally, when $\mathrm{G} 2-\mathrm{H} 2$ approaches (not arriving at) the bottom, the bending of the mean streamlines (in Fig. 12(c)) caused by the upward flow from the base leads to the generation of the vortex pair $C$ and $D$ in Fig. 11(c), the strength of which monotonically reduces with the increase of distance from the obstacle and finally completely vanishes. Stage-III is distinguished by the stabilization of the profile of the mean streamwise vortices, as depicted in Figs. 11 and 12 (f). In this stage, $G 2-H 2$ and $C-D$ have disappeared and the mean streamwise tip vortices $G 1-H 1$ dominate the plane.

Figs. 13-16 demonstrate the six-vortices type evolution of mean streamwise vortices in the wake for case-4 (i.e., Re $=250$ ), being in accordance with the results of Figs. 6 and 7. In fact, case-3 (i.e., $R e=150$ ) shares almost the same transition process, which is omitted here for simplicity. The six-vortices type evolution has some similarities with the dipole-type evolution, but differences do exist between them, as discussed in the following. In the first place, for stage-II, Figs. 13 and 14(b) show that $\mathrm{G} 2-\mathrm{H} 2$ initially emerges near the middle height of the cylinder with the occurrence of the downwash, rather than near the tip region (e.g., Figs. 11 and 12(b)). With the increase of $X$, the strength of $G 2-H 2$ is first enhanced (i.e., in the range of $2 \leq X \leq 6$, Figs. 13(b)-13(d)) and then weakened (i.e., in the range of $6 \leq X \leq 10$, Figs. 13(d)-13(f)), being in accordance with the variation trend of $\mathrm{G} 2-\mathrm{H} 2$ shown in Figs. 11(c)-11(f). In the second place, as the distance from the obstacle increases, the height of $\mathrm{G} 2-\mathrm{H} 2$ is reduced to that of the impingement point $R$ in Fig. 2(d), as confirmed by Figs. 13, 14(e), and 14(f), which is consistent with the aforementioned conclusion that this pair of vortices is eventually convected to the height of the impingement point in the symmetry plane. It is obvious that $\mathrm{G} 2-\mathrm{H} 2$ completely disappears in stage-III because of its rapidly decreasing intensity along the $X$ axis. In the third place, Figs. 13(d)-13(f) also indicate that, during the transition process of $G 2-H 2$, two pairs of counter-rotating vortices (i.e., $C-D$ and $E-F$ ) are generated due to the bending of the mean streamlines in the lower part induced by the upward flow from the base, while only one pair of such vortices (i.e., $C$-D in Figs. 9(a), 9(b), and 11(c)-11(e)) is observed for the quadrupole-type evolution and the dipole-type evolution. Further, with the increase of $X, C-D$ and $E-F$ move laterally away from the centerline and their strength monotonically decreases. In the fourth place, for stage-III at $R e=250$, both mean streamwise tip vortices (i.e., G1-H1) and mean streamwise base vortices-farwake (i.e., $I-J$ ) can be observed in the far-wake region, which suggests that the mean streamwise vortex structure transforms from "six-vortices type" into

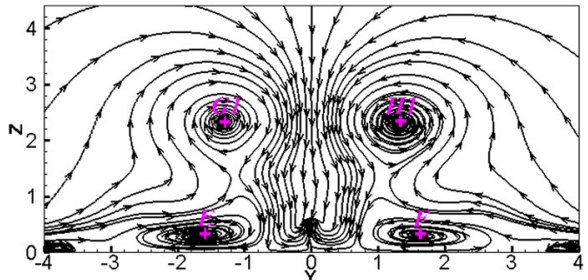

(a) $X=11.0, R e=250$

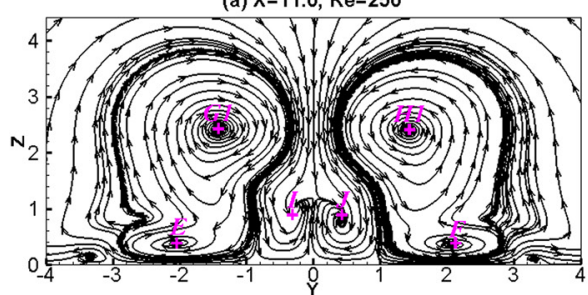

(c) $X=14.0, R e=250$

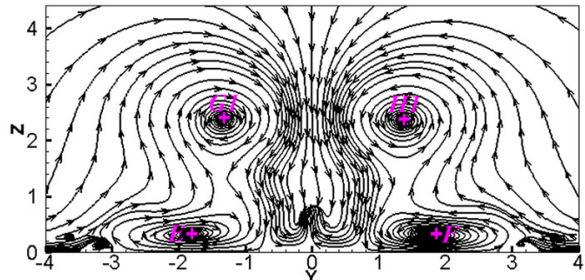

(b) $X=12.0, R e=250$

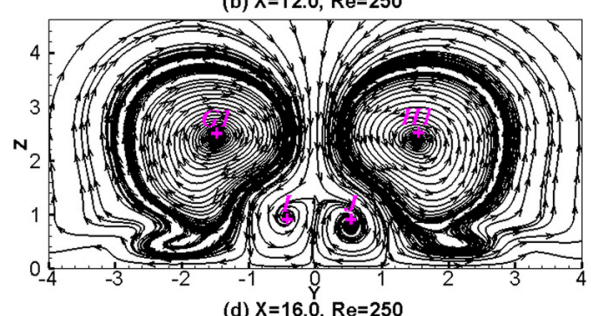

(d) $X=16.0, R e=250$
FIG. 16. The evolution of the timeaveraged streamlines between the near and far wakes at $R e=250$ : Part-2. (a) $X$ $=11.0$; (b) $X=12.0$; (c) $X=14.0$; (d) $X$ $=16.0$. 


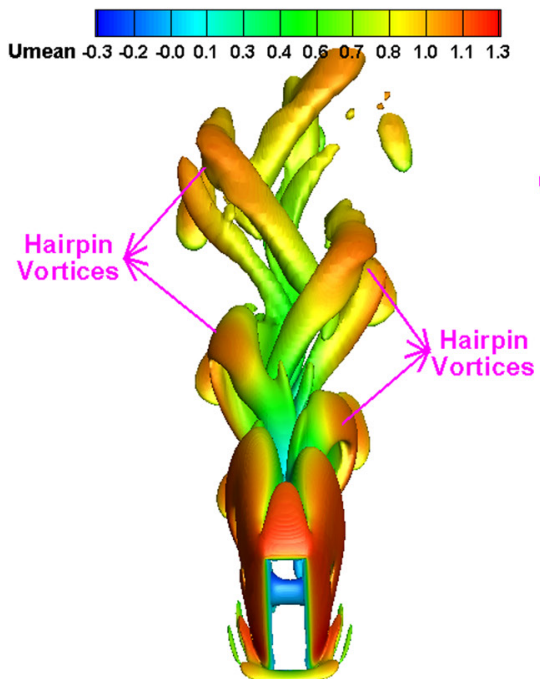

(a) Time-01, $\lambda_{2}=-0.03$

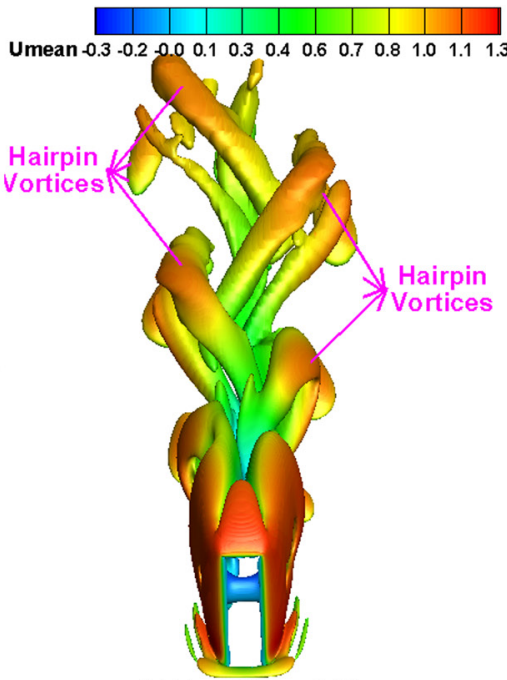

(b) Time- $02, \lambda_{2}=-0.03$

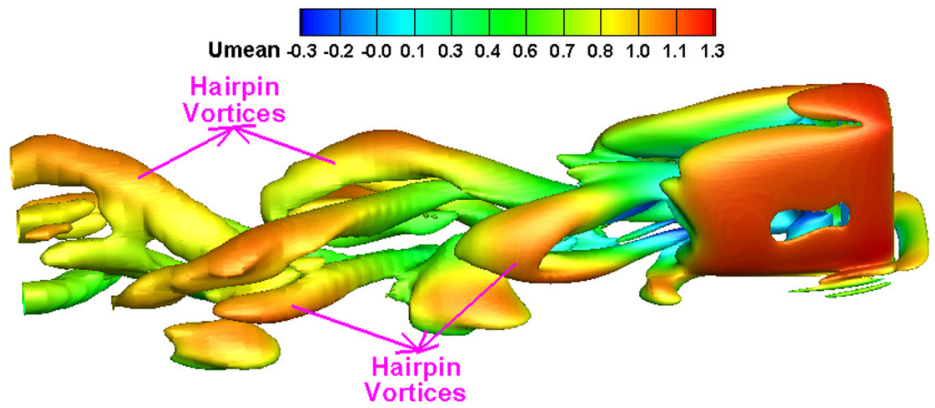

(c) Time- $03, \lambda_{2}=-0.03$

"quadrupole type" as the distance from the cylinder increases at $R e=250$, as presented in Figs. 7, 15, 16(c), and 16(d).

So far, three types of mean streamwise vortex structures have been defined and detailed, namely, the "Quadrupole Type" at $R e=50$ and 100, the "Six-Vortices Type" at $R e=150$ and 250, and the "Dipole Type" at $R e=500$ and 1000 . Correspondingly, three kinds of evolution processes of mean streamwise vortices along the streamwise (i.e., $X$ ) direction have also been explained in detail, which is beneficial to the understanding of the finite-cylinder wake.

\section{Instantaneous spanwise vortices}

This section aims to investigate the three-dimensional spanwise vortex structure behind the cylinder in terms of the $3 \mathrm{D}$ physical model of the vortex-shedding and the $2 \mathrm{D}$

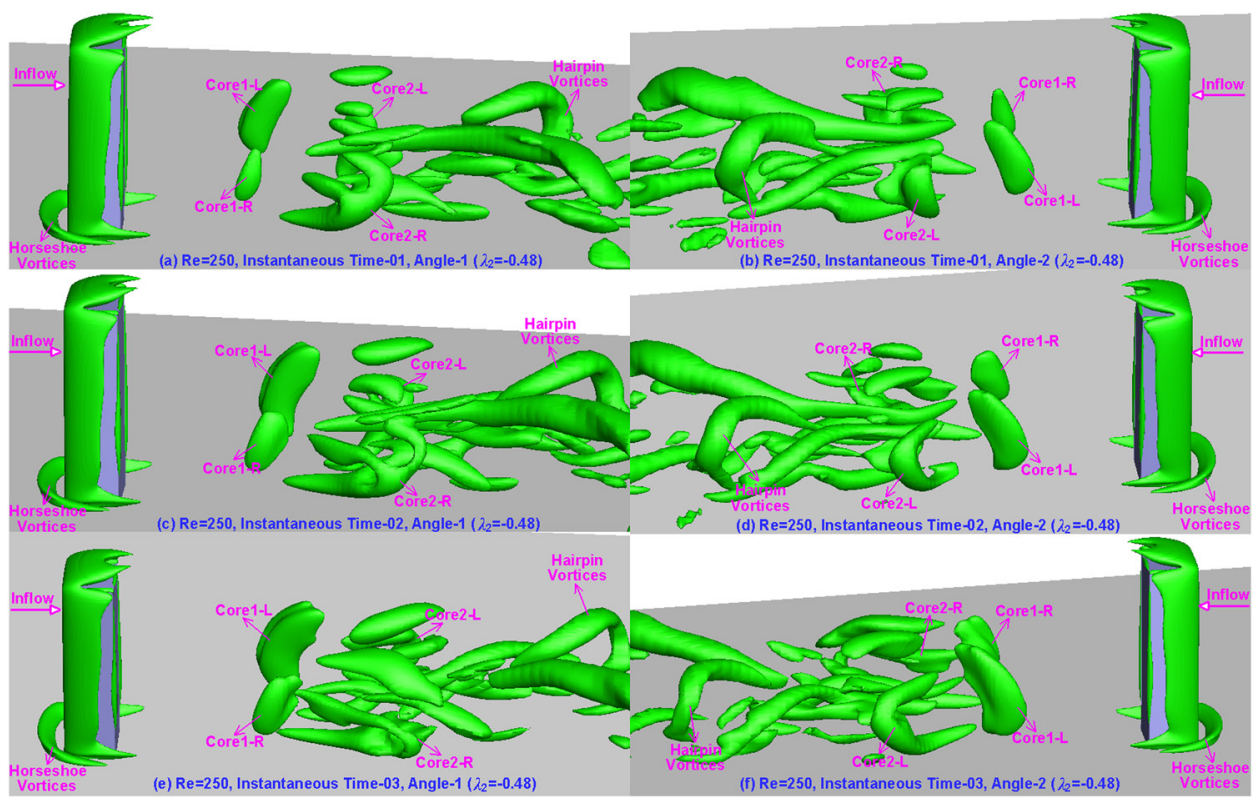

FIG. 18. " $C$ and Reverse- $C$ and Hairpin Vortex Model (Symmetric Shedding)" instantaneous spanwise vortices at $R e=250\left(\lambda_{2}=-0.48\right):((a)$ and (b)) time-01; ((c) and (d)) time-02; ((e) and (f)) time-03. 


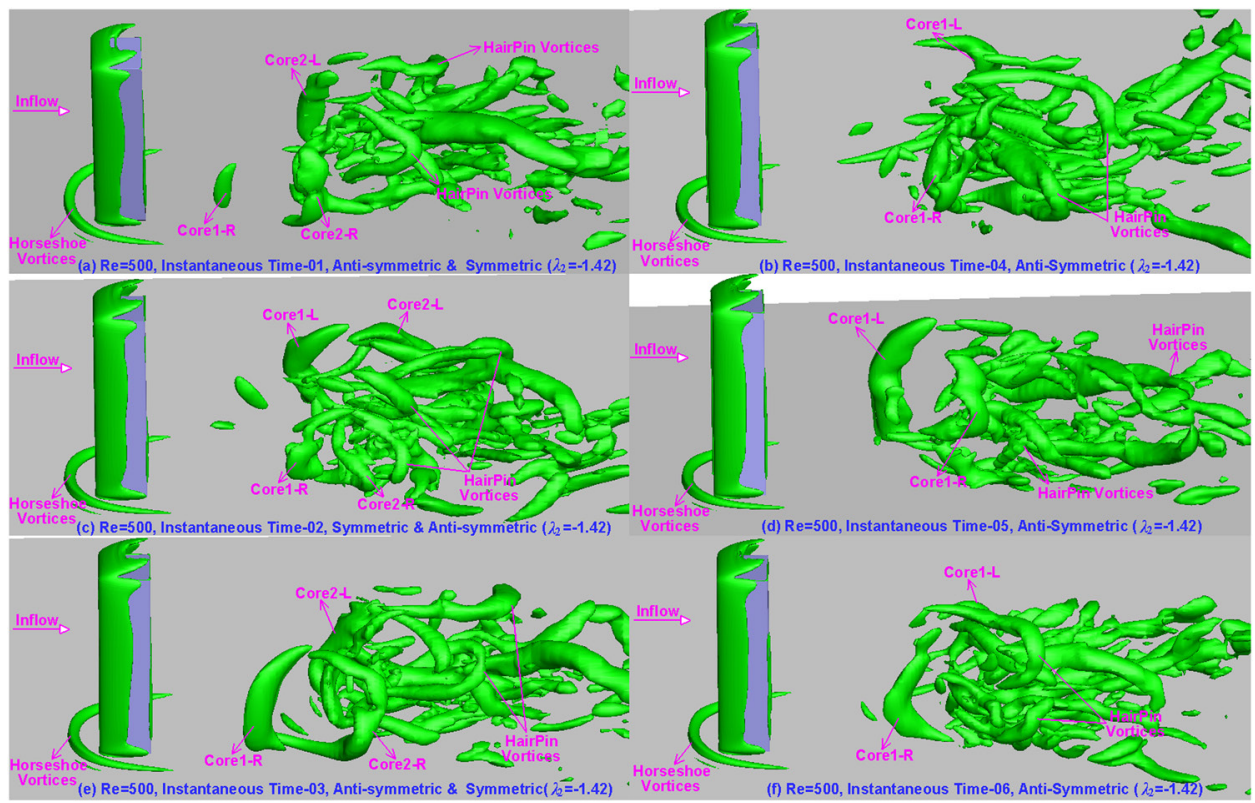

FIG. 19. " $C$ and Reverse- $C$ and Hairpin Vortex Model (Symmetric/Antisymmetric Shedding)" instantaneous spanwise vortices at $R e=500$ $\left(\lambda_{2}=-1.42\right)$ : ((a), (c), and (e)) symmetric and anti-symmetric; ((b), (d), and (f)) anti-symmetric.

vorticity contours in various horizontal planes at some characteristic moments.

\section{Physical model of vortex-shedding}

When it comes to the identification of vortex cores, the aforementioned $\lambda_{2}$ criterion $^{55}$ has proved to be more effective than other common indicators of vortices (e.g., pressure minimum, closed or spiraling streamlines and pathlines, and iso-vorticity surfaces) and is therefore widely used in the literature. ${ }^{3,16,19,44,45}$ As described in Table II, the wake flow is steady and no vortex-shedding can be detected at $R e=50$ and $R e=100$. Besides, Figs. 17-20 manifest that three kinds of vortex-shedding models can be observed with the increase of $R e$, namely, "Hairpin Vortex Model" at Re $=150\left(\lambda_{2}=-0.03\right.$, indicating that the vortex-shedding phenomenon is very weak at this $R e$ ), " $C$ and Reverse-C and Hairpin Vortex Model (Symmetric Shedding)" at Re $=250$ $\left(\lambda_{2}=-0.48\right)$, and " $C$ and Reverse- $C$ and Hairpin Vortex Model (Symmetric/Antisymmetric Shedding)" at $R e=500\left(\lambda_{2}\right.$ $=-1.42)$ and $R e=1000\left(\lambda_{2}=-2.48\right)$. Considering that the "Hairpin Vortex Model" has already been analyzed in detail by Dousset and Potherat ${ }^{19}(A R=4, R e=200)$ and Saha ${ }^{16}$ $(A R=3, R e=250)$, the remaining part of this section will focus on the analysis of the two newly proposed models, namely, " $C$ and Reverse- $C$ and Hairpin Vortex Model (Symmetric Shedding)" and "C and Reverse-C and Hairpin Vortex Model (Symmetric/Antisymmetric Shedding)," together with their comparison with several existing models presented in the literature.

First, as confirmed in Figs. 18-20, the " $C$ and Reverse$C$ and Hairpin Vortex Model" does not contain the transverse instantaneous tip vortex, the streamwise instantaneous tip vortices, the mean streamwise tip vortices, and the mean streamwise base vortices. The reasons will be given in the

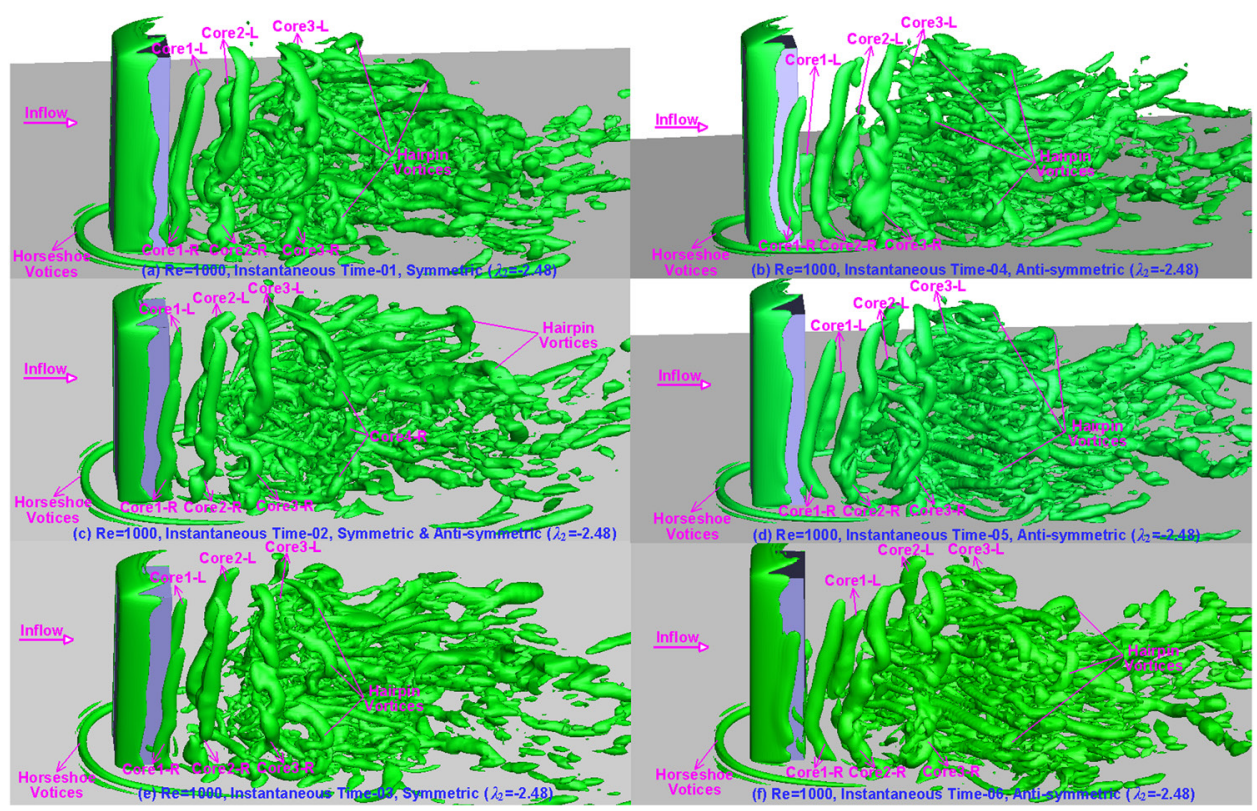

FIG. 20. " $C$ and Reverse-C and Hairpin Vortex Model (Symmetric/Antisymmetric Shedding)" instantaneous spanwise vortices at $R e=1000$ $\left(\lambda_{2}=-2.48\right)$ : ((a) and (e)) symmetric; (c) symmetric and anti-symmetric; ((b), (d), and (f)) anti-symmetric. 
following. The transverse instantaneous tip vortex has been observed by Saeedi et al. ${ }^{12}$ and Hwang and Yang, ${ }^{18}$ however, no such kind of vortex-shedding is observed in this study, which may be attributed to the much lower $R e$ values considered here. The streamwise instantaneous tip vortices are identified by investigating the vorticity field, being consistent with the observations of Roh and Park, ${ }^{38}$ Hain et al.,${ }^{40} \mathrm{Kra}-$ jnovic, ${ }^{5}$ and Park and Lee, ${ }^{17,39}$ but, this type of vortex quickly vanishes along the streamwise direction (being visible only when $X / D \approx 1-3$ in Figs. 11 and 13) and its strength is relatively very low when compared with that of the laterally shed spanwise vortices. Consequently, no independent vortex structure corresponding to this kind of vortex is observed in Figs. 18-20, which essentially depict large-scale vortex structures. Besides, although the mean streamwise tip/base vortices, which are, respectively, induced by the downwash from the cylinder tip and the upwash near the bottom wall, serve as the constituent parts of several existing vortex models, they can only be observed at some distance from cylinder ${ }^{1,2,21,43}$ and are meaningful only for the time-averaged flow field. Therefore, the two types of mean streamwise vortices cannot be captured as well in Figs. 18-20.

Second, in many previous studies, ${ }^{23,24,43,46}$ it is believed that a critical $A R$ value (i.e., $A R_{c} \approx 2-5$ ) exists when it comes to the pattern of the spanwise vortex. In other words, for $A R$ $>A R_{c}$, the antisymmetric Karman vortex-shedding occurs along most of the span, but, for $A R<A R_{c}$, the flow near the free-end suppresses the Karman vortex and eventually leads to a symmetric arch-type vortex along the span in the near-wake. However, our numerical results manifest that the type of the spanwise vortex is also significantly influenced by $R e$ (at least for the $R e$ range considered here), and, further, even for prescribed $A R$ and $R e$ values, both the symmetric and asymmetric vortex-shedding types are possible, which agrees well with the experimental observation of Wang and Zhou ${ }^{1}$ and Wang et $a l .{ }^{25}$ As indicated in Fig. 18, only the symmetric spanwise vortex is observed at $R e=250$. However, Figs. 19 and 20 illustrate that, for $R e=500$ and $R e=1000$, both the symmetric and
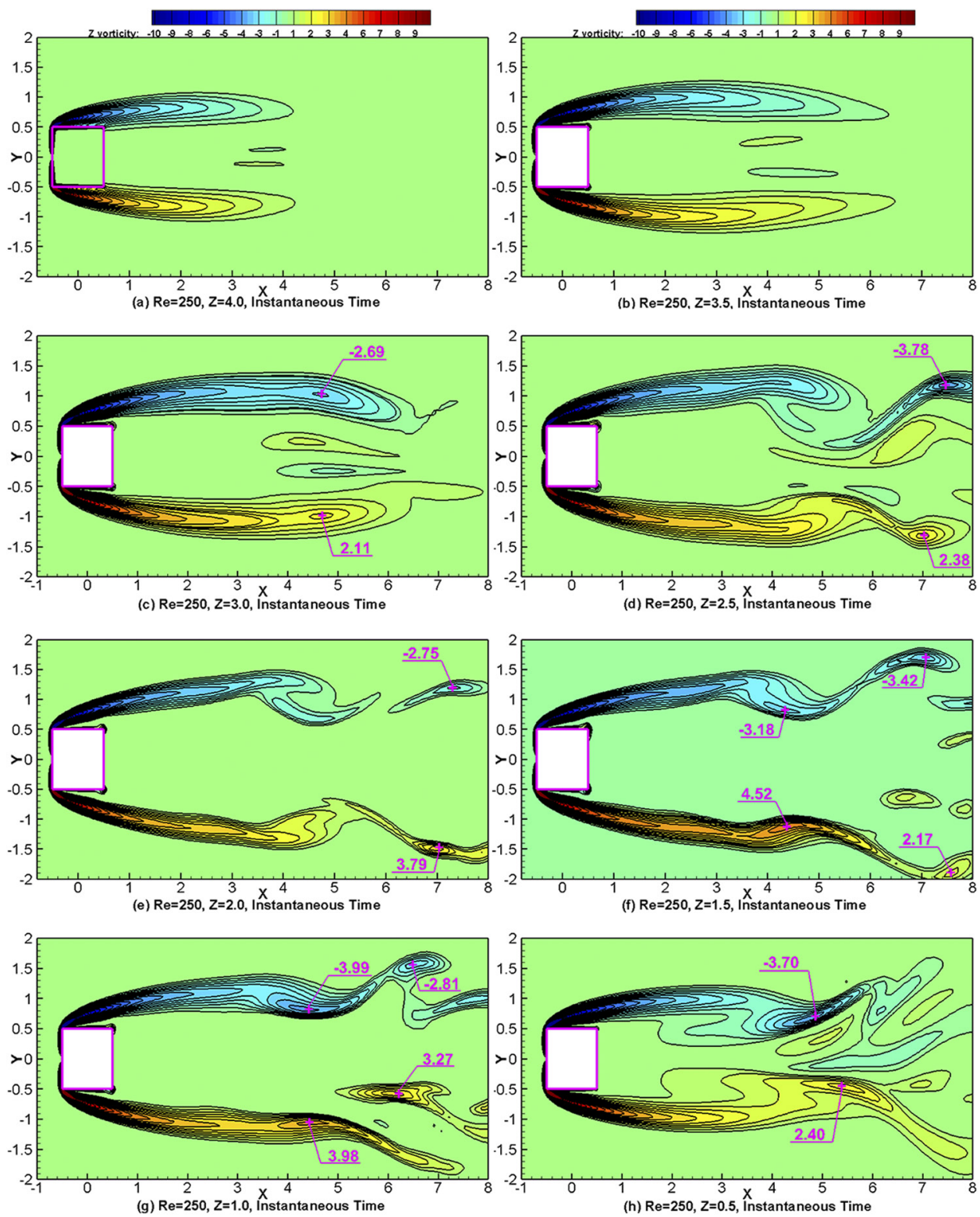

FIG. 21. The 2D vorticity contours in various horizontal planes at an instantaneous time for $\operatorname{Re}=250(Z=4.0-0.5)$. 


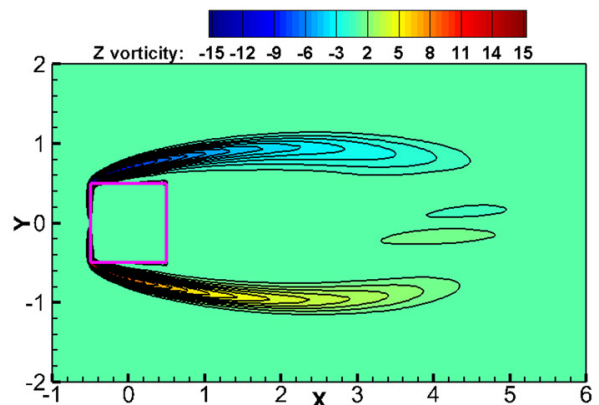

(a) $\mathrm{Re}=500, Z=4.0$, Instantaneous Time, No Vortex-shedding

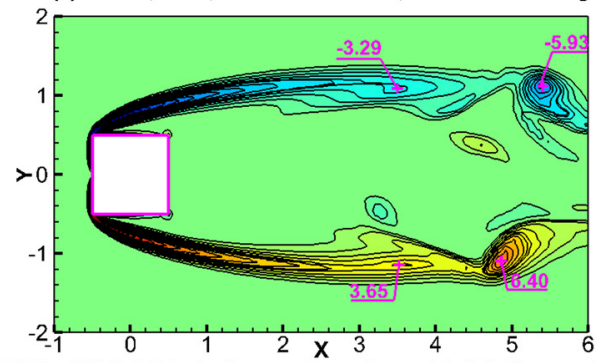

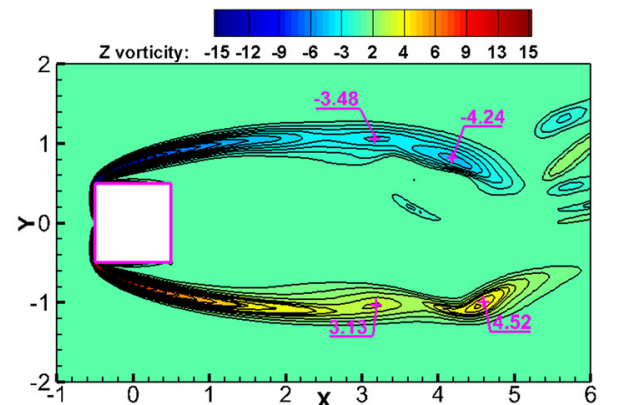

(b) $R e=500, z=3.5$, Instantaneous Time, Symmetric \& Anti-symmetric

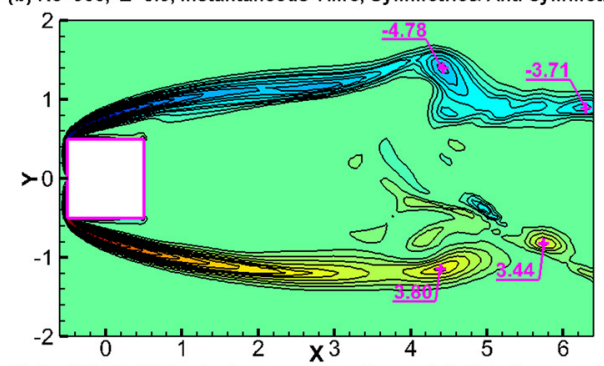

FIG. 22. The 2D vorticity contours in various horizontal planes at an instantaneous time for $\operatorname{Re}=500(Z=4.0-2.5)$.

(c) $R e=500, Z=3.0$, Instantaneous Time, Symmetric \& Anti-symmetric (d) $R e=500, Z=2.5$, Instantaneous Time, Symmetric \& Anti-symmetric

antisymmetric vortex structures are present in the wake. Special attentions are needed for Figs. 19(a), 19(c), 19(e), and 20(c), which reveal that, at some characteristic moments, the symmetric and antisymmetric spanwise vortex cores coexist in the wake because of the random occurrence of these two types of vortex-shedding.

Third, it is very clear from Figs. 18-20 that, whether for the symmetric or for the anti-symmetric arrangement, in the early stage of the spanwise vortex development, its one/two "legs" which are shed from the two sides of the cylinder exhibit the " $C$ " shape, which means that both the upper and the lower parts of the vortex cores are inclined toward the downstream direction. Nevertheless, with the increase of the distance from the cylinder, the profile of the spanwise vortices will be transformed into the "Reverse- $C$ " shape, which is characterized by the inclination of the two ends of the vortex rolls towards the upstream direction and their bending towards the center plane. Moreover, in the further downstream region, the "Reverse- $C$ " type of vortices transits to the "Hairpin" type of vortices with the fragmentation of large pieces of vortex structure because of
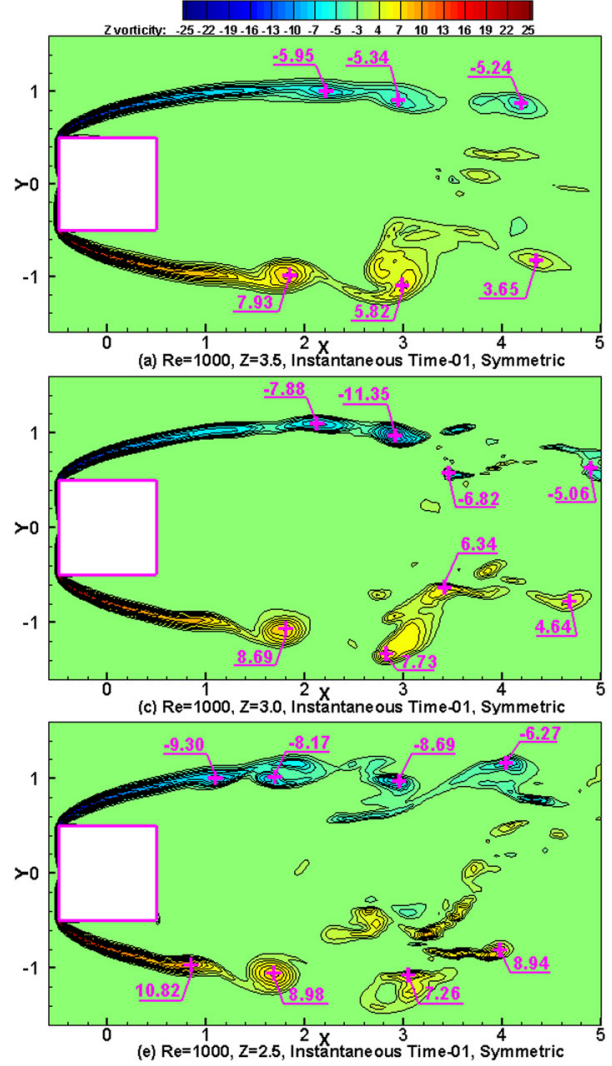

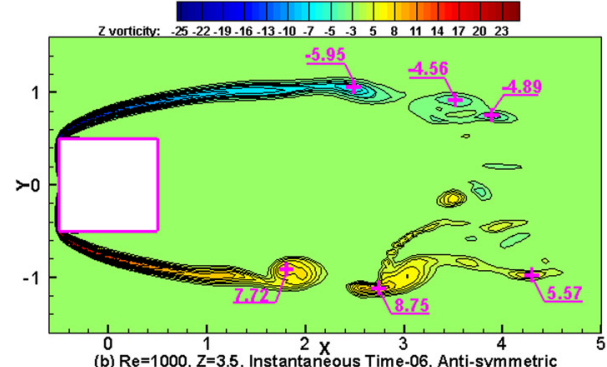

0
(b) $\operatorname{Re}=1000, \mathrm{Z}=3.5$, Instantaneous Time-06, Anti-symmetric

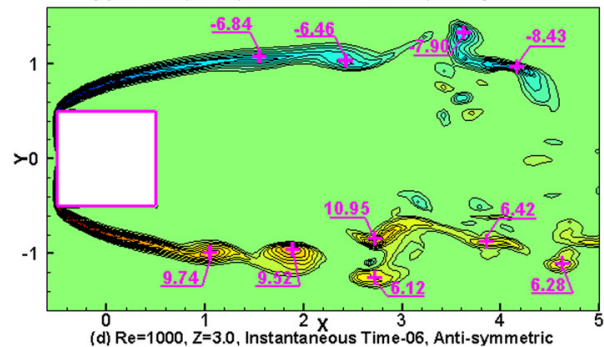

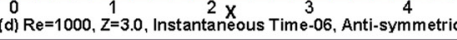

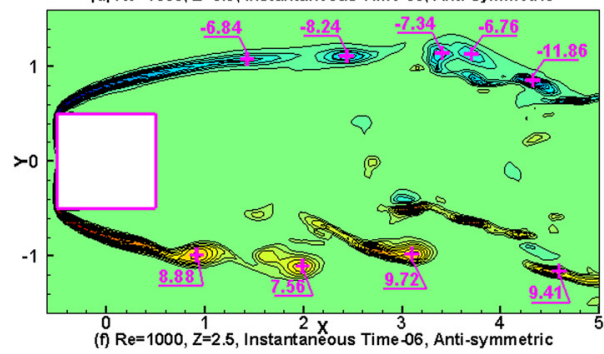

FIG. 23. The 2D vorticity contours in various horizontal planes at two characteristic moments for $R e=1000(Z=3.5-$ 2.5): ((a), (c), and (e)) symmetric, time01; ((b), (d), and (f)) anti-symmetric, time-06. 
the turbulence induced by the interactions between the downwash, the shear layer, and the boundary layer. It is apparent that, with the increase of $R e$, the degree of the fragmentation is enhanced, and the location, at which the fragmentation occurs, moves toward the cylinder (viz., with a smaller $X$ value). Besides, relative to $R e=9300$ considered in "Wang's model,"1 the $R e$ values (i.e., $R e \leq 1000$ ) employed in this study are much smaller. This leads to much weaker instantaneous transverse tip vortices, and consequently the connection line near the tip region (existing in "Wang's Model," as displayed by Fig. 11 in Ref. 1) is absent in the present model. Additionally, Figs. 18-20 also confirm that the length of the spanwise vortices (in the vertical direction) monotonically increases with the increase of $R e$ because the free-end has a stronger suppression effect on the spanwise vortex-shedding near the tip region at low $R e$ values, and at the same time the increase of the thickness of the boundary layer leads to the enhanced inhibition of the spanwise vortices near the base region.

\section{Two-dimensional vorticity contours}

In order to further validate the " $C$ and Reverse- $C$ and Hairpin Vortex Model (Symmetric Shedding)," Fig. 21 quantitatively shows the instantaneous 2D vorticity contours in various horizontal planes along the span at a typical moment for case-4 (i.e., $R e=250$ ). The same color scale is adopted, and the centers associated with negative or positive vorticity concentrations are marked with "+" in these figures. Being consistent with the vortex structure presented in Fig. 18, only symmetric spanwise vortices are observed in this scenario, and the vortex-shedding phenomenon occurs within the extent of $Z / D \leq 3.0$. In other words because of the suppression of the free-end, no vortex-shedding can be identified near the tip region (e.g., $Z / D=4.0$ and $Z / D=3.5$ ), as confirmed by Figs. 21(a) and 21(b). However, the spanwise vortex-shedding becomes possible at the height of $Z / D \approx 3.0$, and its strength continuously increases with the increase of the distance from the tip and reaches the maximum value at about $Z / D \approx 1.5$.

In order to further demonstrate the " $C$ and Reverse- $C$ and Hairpin Vortex Model (Symmetric/Antisymmetric Shedding)," Figs. 22-24 show the instantaneous 2D vorticity contours of spanwise vortices in various horizontal planes at some typical moments for case-5 $(R e=500)$ and case-6 $(R e=1000)$. Especially, the moment depicted by Fig. 22 actually corresponds to that illustrated in Fig. 19(c), in which one pair of symmetric " $C$-Shape" spanwise vortices and another pair of anti-symmetric "Reverse-C Shape" vortices coexist in the wake. Figs. 23(a), 23(c), 23(e), 24(a), 24(c), and 24(e), where several pairs of symmetric vortex cores on different sections along the span are observed, share the same moment as that of Fig. 20(a) (i.e., instantaneous time-01 in Fig. 20). However, Figs. 23(b), 23(d), 23(f), 24(b), 24(d), and 24(f), where the anti-symmetric vortices in various horizontal planes are observed, present the contours of $\omega_{z}$ at the same moment as that of Fig. 20(f) (i.e., instantaneous time-06 in Fig. 20). From these figures, it can be concluded that, with the increase of $R e$, the distance from the cylinder at which the vortexshedding structure appears is reduced. This is attributed to the enhanced shear layers from the leading edges of the cylinder at large $R e$ values. In addition, Figs. 21(b), 22(b), 23(a), and 23(b), respectively, show that, on the $Z / D=3.5$ plane, no vortex-shedding is observed at $R e=250$, relatively
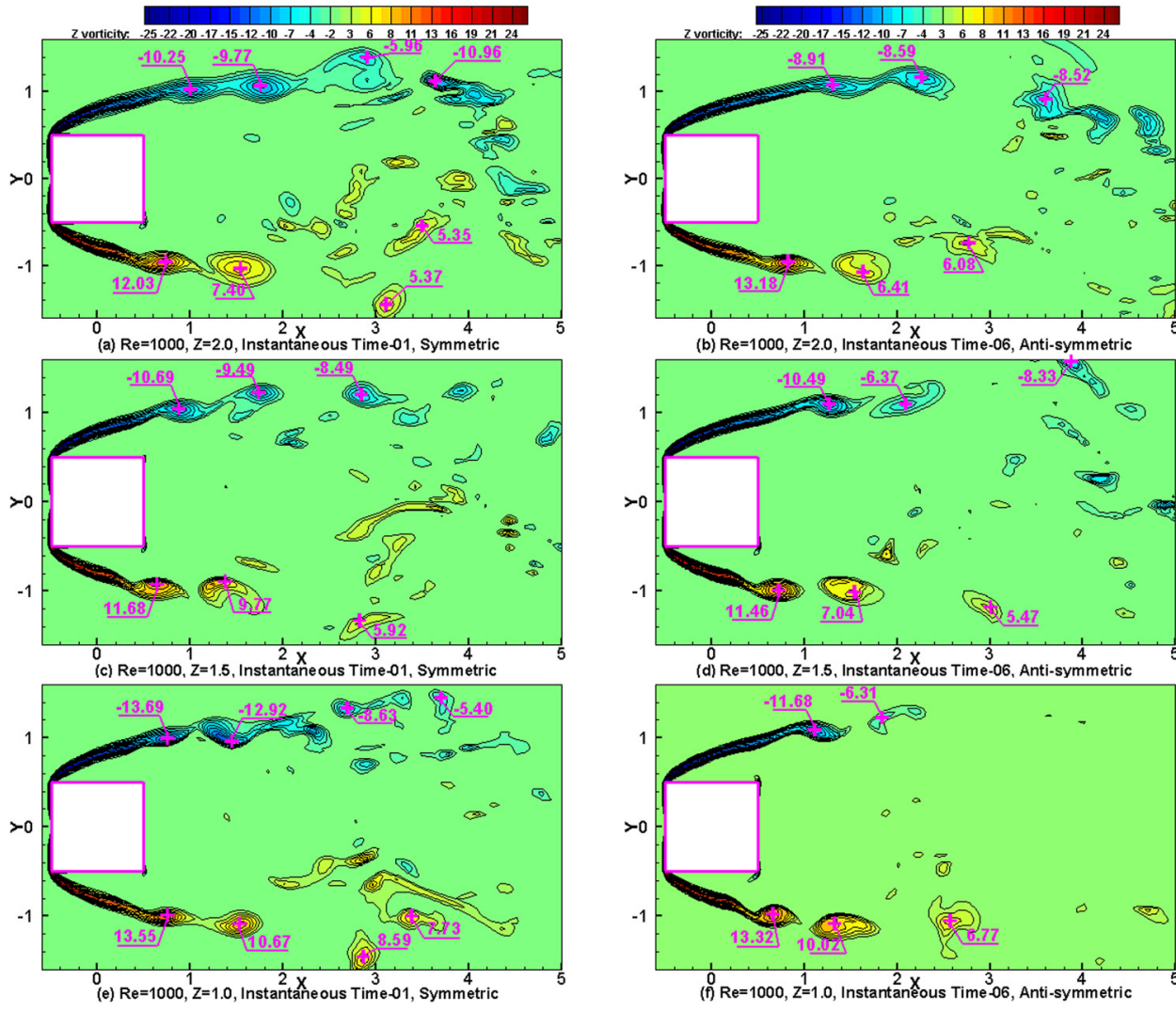

FIG. 24. The 2D vorticity contours in various horizontal planes at two characteristic moments for $\operatorname{Re}=1000(Z=2.0$ 1.0): ((a), (c), and (e)) symmetric, time01; ((b), (d), and (f)) anti-symmetric, time-06. 
weak symmetric/anti-symmetric vortex-shedding appears at $R e=500$, and relatively strong symmetric/anti-symmetric spanwise vortices are observed at $R e=1000$, which supports the conclusion once again that the length of the spanwise vortex in the vertical direction is increased with the increase of $R e$ because of the relatively weak suppression of the free-end at large $R e$ values, as discussed in Subsection IV C 1.

\section{CONCLUSIONS}

Flow past a finite square cylinder $(A R=4)$ is studied at six $R e$ values $(R e=50,100,150,250,500$, and 1000). The focus is to examine the influence of $R e$ on three aspects of the flow, namely, the time-averaged velocity and pressure fields, the mean streamwise vortices, and the vortex-shedding model of instantaneous spanwise vortices. The main findings include the following:

1. Although in the literature it was found that the mean streamwise vortex pattern is influenced by the $A R$ value, this study indicates that the formation of mean streamwise vortices is also affected by $R e$. For the fixed $A R=4$, three types of mean streamwise vortices have been observed and analyzed in detail, namely, "Quadrupole Type" at Re $=50$ and $R e=100$, "Six-Vortices Type" at $R e=150$ and $R e=250$, and "Dipole Type" at $\operatorname{Re}=500$ and $R e=1000$.
Especially, to the best of our knowledge, it is the first time that the "Six-Vortices Type" mean streamwise vortices are reported, which is considered as a transitional structure between the other two types.

2. In terms of instantaneous spanwise vortices, three kinds of vortex-shedding models are observed, namely, "Hairpin Vortex Model" at $R e=150, " C$ and Reverse- $C$ and Hairpin Vortex Model (Symmetric Shedding)" at Re $=250$, and " $C$ and Reverse- $C$ and Hairpin Vortex Model (Symmetric/Antisymmetric Shedding)" at $R e=500$ and $R e$ $=1000$. The newly defined " $C$ and Reverse- $C$ and Hairpin Vortex Model" shares some similarities with "Wang's Model." However, the two models do differ in some aspects, such as (in the present model) the connection line near the tip region is absent, in the early stage of the formation of the spanwise vortex the shape of the vortex cores is actually "C Shape," and in the further downstream region the "Reverse-C Shape" transforms into the "Hairpin Vortex Shape" due to the fragmentation of large pieces of vortex structure.

3. By visualizing the flow through the mean streamlines and the mean vorticity contours in various streamwise planes, this paper presents the detailed evolution process of mean streamwise vortices between the near and far wakes, which has not been reported in the

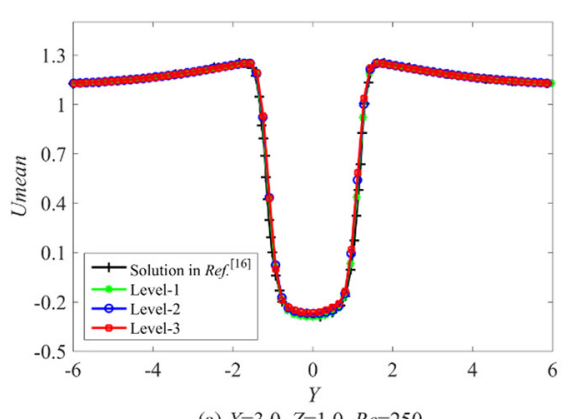

(a) $X=3.0, Z=1.0, R e=250$
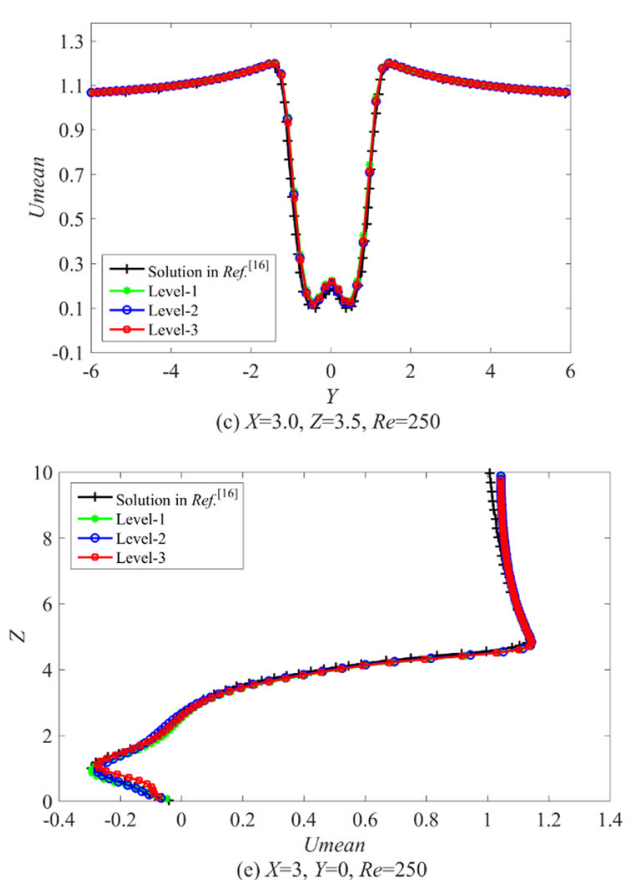

(e) $X=3, Y=0, R e=250$

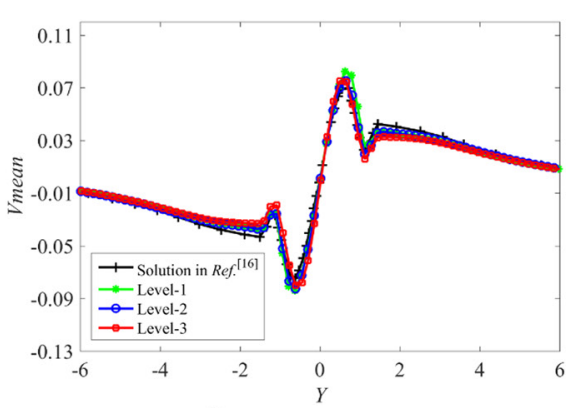

(b) $X=3.0, Z=1.0, R e=250$
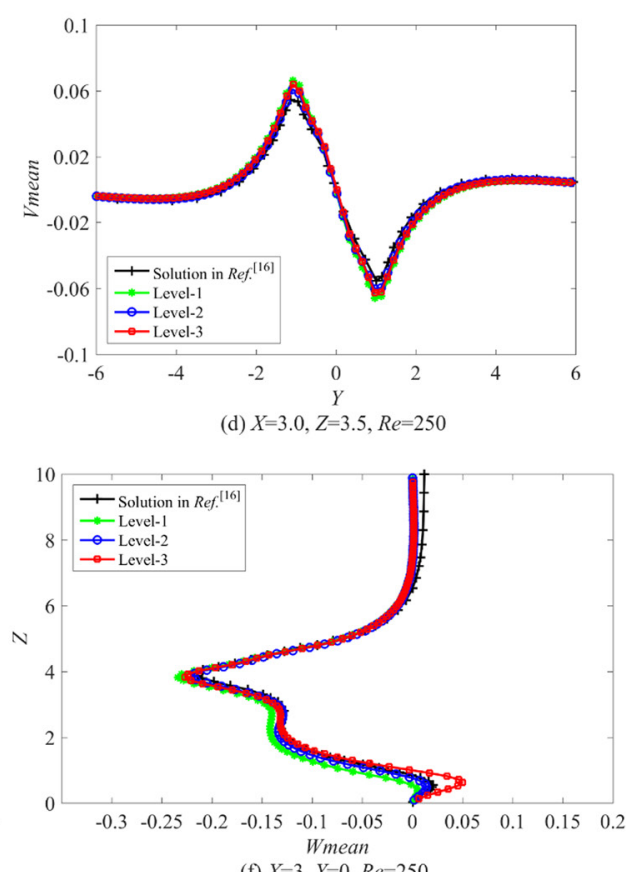

(f) $X=3, Y=0, R e=250$
FIG. 25. Profiles of various mean velocity components in the section of $X$ $=3.0$ at $R e=250$. (a) Umean, $Z=1.0$; (b) vmean, $Z=1.0$; (c) umean, $Z=3.5$; (d) vmean, $Z=3.5$; (e) umean, $Y=0$; (f) wmean, $Y=0$. 
literature. Further, three kinds of transition processes are defined and explained, namely, quadrupole-type evolution, dipole-type evolution, and six-vortices type evolution.

4. Serving as an extension of the conclusions of previous research, it is put forward that the mean streamwise vortex structure is dependent on if the downwash from the cylinder tip can reach the bottom wall, namely, whether or not the downwash in the upper part and the upwash in the lower part coexist in the symmetry plane (depending on the combined influence of $R e, A R$, and $\delta / D)$. When the downwash can reach the bottom wall in the symmetry plane, only a pair of counter-rotating mean streamwise tip vortices (i.e., "Dipole Type") is observed in the wake, but, when the downwash fails to reach the bottom wall, either "Quadrupole Type" or "SixVortices Type" mean vortex structure is formed in the wake.

\section{ACKNOWLEDGMENTS}

The authors wish to acknowledge the financial support provided by Australian Research Council Discovery Grant (Project No. DP150104644) and the computational resources provided by the Pawsey Supercomputing Centre with fund- ing from the Australian Government and the Government of Western Australia. The leading author gratefully acknowledges the valuable suggestions from Dr. Scott Draper at the University of Western Australia in the preparation of this paper.

\section{APPENDIX: THE GRID CONVERGENCE}

This part presents a quantitative comparison of the profiles of three mean velocity components in different sections achieved by making use of the four newly designed nonuniform structured meshes (i.e., the level-1, level-2, level-3, and level-4 grids detailed in Table I) with the purpose of demonstrating the grid-independency of the numerical results.

Figs. 25 and 26 show the profiles of various velocity components in two streamwise planes (i.e., $X=3.0$ and $X=7.0$ ) at a Reynolds number of 250 , together with the numerical results extracted from Ref. 16. Obviously, the numerical results obtained by using level-2 and level-3 agree very well with the existing data, except for the umean profile along the junction line of $X=7.0$ and $Z=3.5$ (viz., Fig. 26(c)), but even in this circumstance the grid convergence is very well realized through the newly designed three grid levels. Overall, these figures have demonstrated that level-2 and level-3 are fine enough to obtain the grid-independent solution for

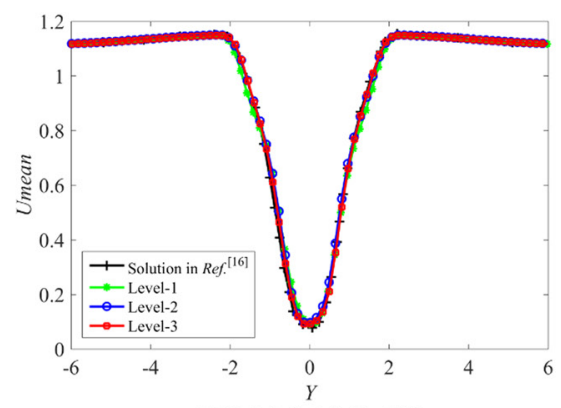

(a) $X=7.0, Z=1.0, R e=250$

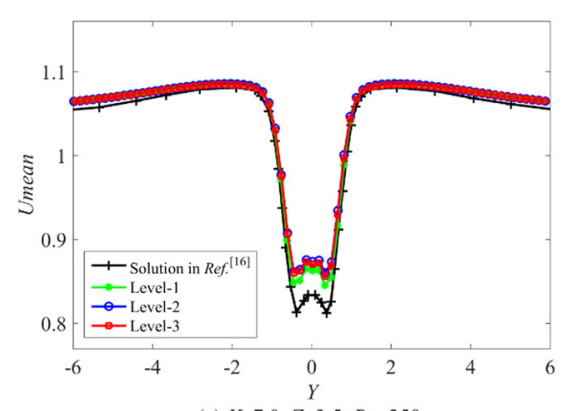

(c) $X=7.0, Z=3.5, R e=250$

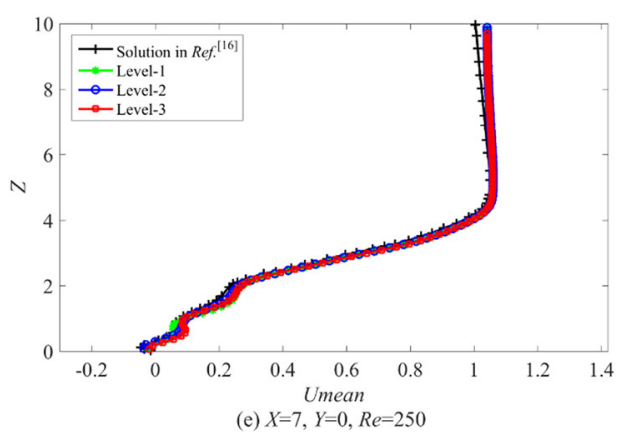

(e) $X=7, Y=0, R e=250$

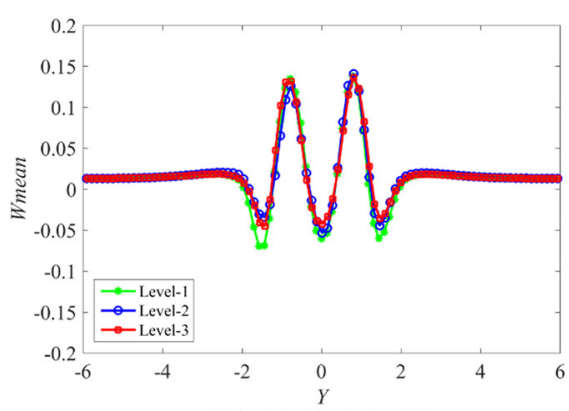

(b) $X=7.0, Z=1.0, R e=250$

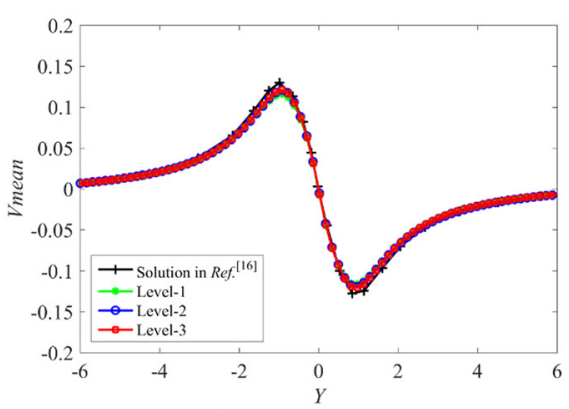

(d) $X=7.0, Z=3.5, R e=250$

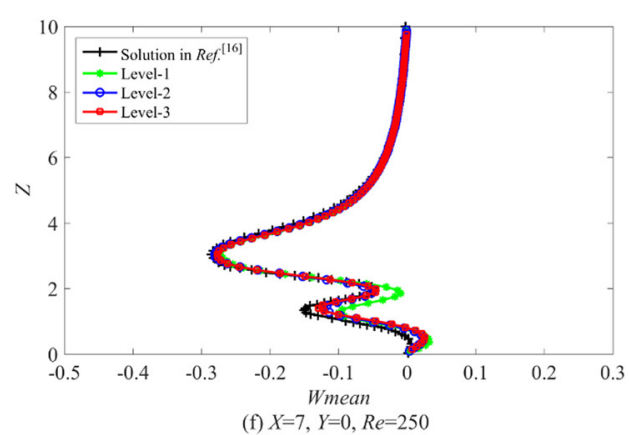

FIG. 26. Profiles of various mean velocity components in the section of $X$ $=7.0$ at $R e=250$. (a) Umean, $Z=1.0$; (b) wmean, $Z=1.0$; (c) umean, $Z=3.5$; (d) vmean, $Z=3.5$; (e) umean, $Y=0$; (f) wmean, $Y=0$. 


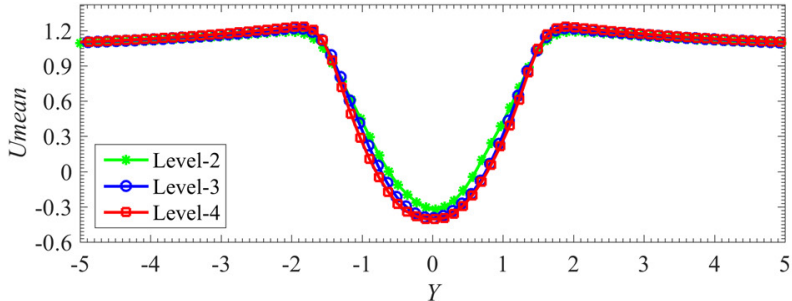

(a) $X=3.0, Z=1.0(R e=1000)$

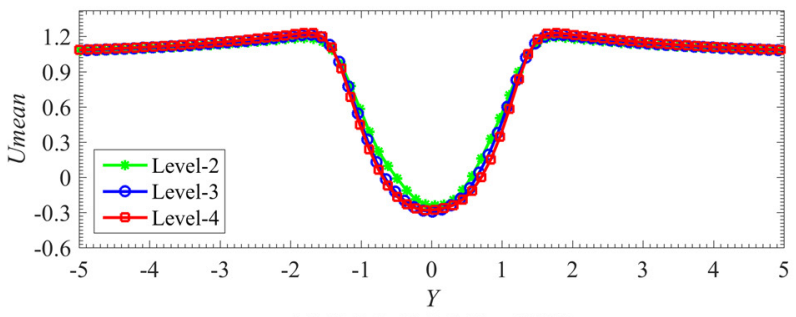

(c) $X=3.0, Z=2.0(R e=1000)$

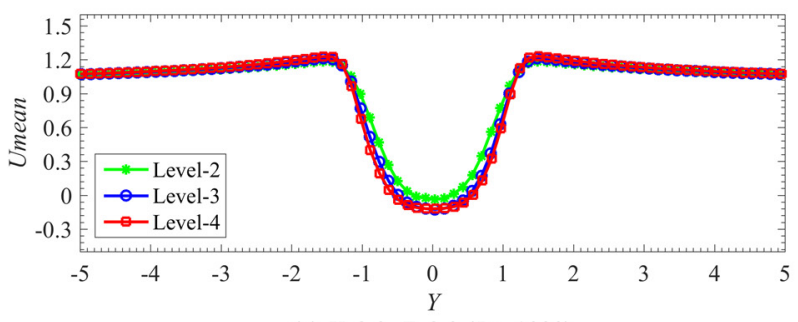

(e) $X=3.0, Z=3.0(R e=1000)$

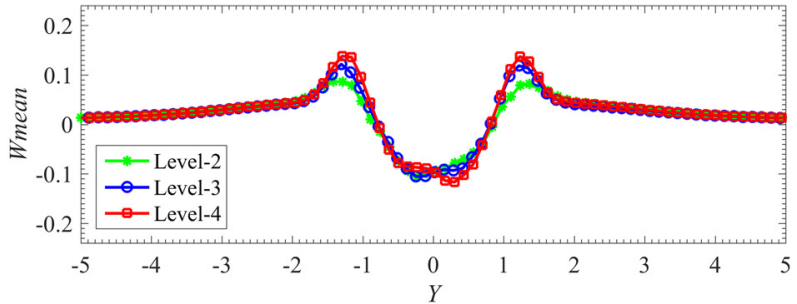

(b) $X=3.0, Z=1.0(R e=1000)$

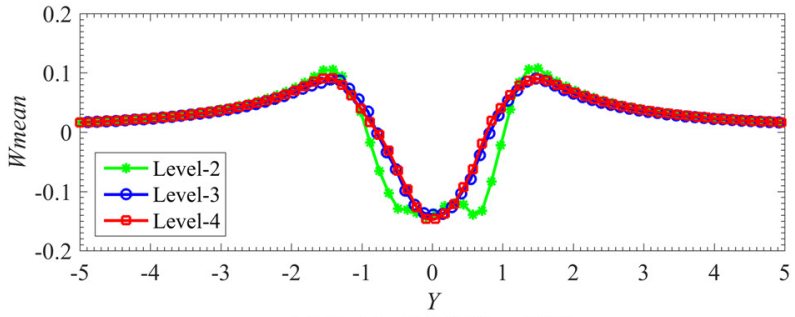

(d) $X=3.0, Z=2.0(R e=1000)$

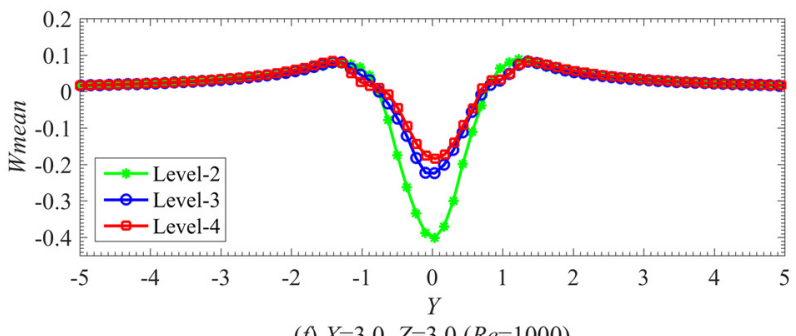

(f) $X=3.0, Z=3.0(R e=1000)$

FIG. 27. Profiles of various mean velocity components in the section of $X=3.0$ at $\operatorname{Re}=1000$. (a) Umean, $Z=1.0 ;$ (b) wmean, $Z=1.0 ;$ (c) umean, $Z=2.0$; (d) wmean, $Z=2.0$; (e) umean, $Z=3.0$; (f) wmean, $Z=3.0$.

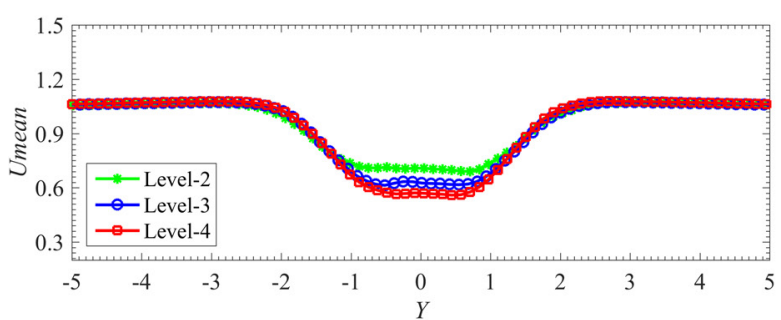

(a) $X=7.0, Z=2.0(R e=1000)$

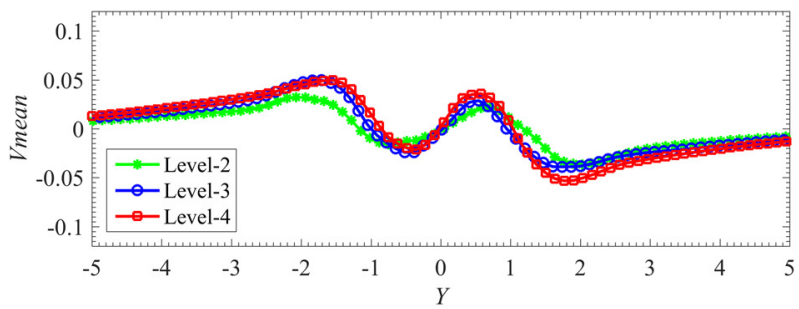

(c) $X=7.0, Z=2.0(R e=1000)$

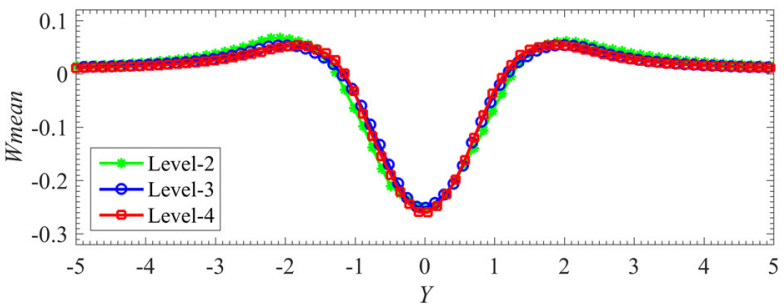

(e) $X=7.0, Z=2.0(R e=1000)$

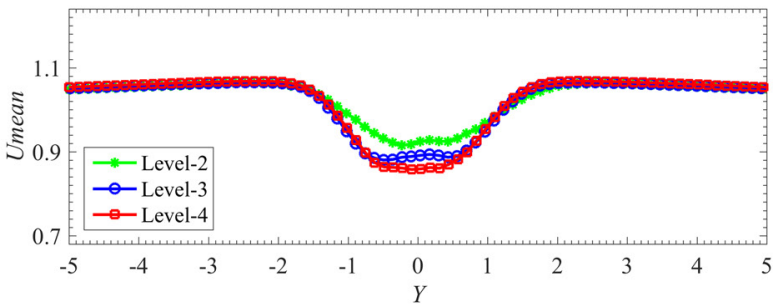

(b) $X=7.0, Z=3.0(R e=1000)$

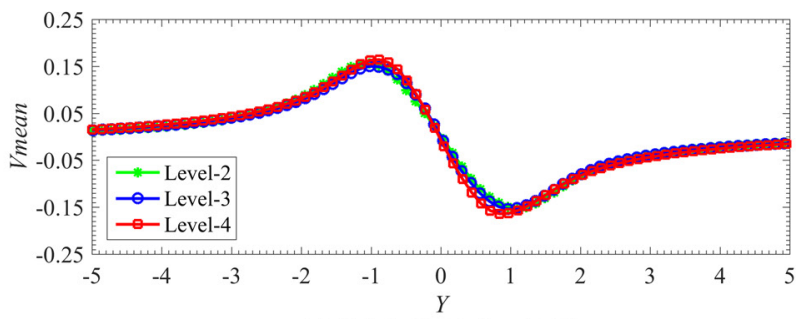

(d) $X=7.0, Z=3.0(R e=1000)$

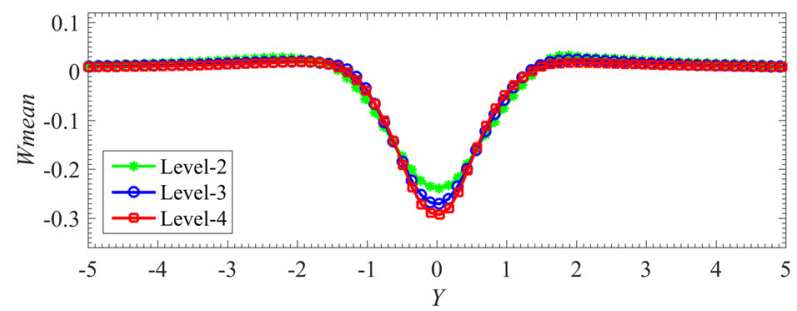

(f) $X=7.0, Z=3.0(R e=1000)$

FIG. 28. Profiles of various mean velocity components in the section of $X=7.0$ at $R e=1000$. (a) Umean, $Z=2.0$; (c) vmean, $Z=2.0$; (e) wmean, $Z=2.0$; (b) umean, $Z=3.0$; (d) vmean, $Z=3.0$; (f) wmean, $Z=3.0$. 
case-4 (i.e., $R e=250$ ), and actually the level-3 mesh is ultimately utilized to carry out the simulation of case-1 (i.e., $R e=50$ ), case-2 (i.e., $R e=100$ ), case-3 (i.e., $R e=150$ ), case-4 (i.e., $R e=250$ ), and case-5 (i.e., $R e=500$ ) in this study in order to improve the spatial accuracy as much as possible.

A further validation is still necessary for the purpose of verifying the grid independency at the largest $R e$ number of this study (i.e., case-6 $(R e=1000)$ ), which imposes higher demands on the grid resolution. Figs. 27 and 28 show the profiles of various velocity components in two streamwise sections (i.e., $X=3.0$ and $X=7.0$ ), and it can be concluded that, by making use of level-3 and level-4, the grid convergence has been well realized. It should be emphasized that, for case-6, the finest mesh (i.e., level-4, $303 \times 228 \times 202$ grid points in $X, Y$, and $Z$ directions, respectively), being approximately 5 times finer than Saha's middle level (i.e., $177 \times 136 \times 120$ ), is selected to carry out the simulation in order to improve the spatial accuracy as much as possible.

${ }^{1}$ H. F. Wang and Y. Zhou, "The finite-length square cylinder near wake," J. Fluid Mech. 638, 453-490 (2009).

${ }^{2}$ D. Sumner and J. L. Heseltine, "Tip vortex structure for a circular cylinder with a free end," J. Wind. Eng. Ind. Aerod. 96(6), 1185-1196 (2008).

${ }^{3}$ J. A. Bourgeois, P. Sattari, and R. J. Martinuzzi, "Alternating halfloop shedding in the turbulent wake of a finite surface-mounted square cylinder with a thin boundary layer," Phys. Fluids 23(9), 095101 (2011).

${ }^{4}$ T. Kawamura, M. Hiwada, T. Hibino, I. Mabuchi, and M. Kumada, "Flow around a finite circular cylinder on a flat plate: Cylinder height greater than turbulent boundary layer thickness," Bull. JSME 27(232), 2142-2151 (1984).

${ }^{5}$ S. Krajnovic, "Flow around a tall finite cylinder explored by large eddy simulation," J. Fluid Mech. 676, 294-317 (2011).

${ }^{6}$ S. Draper, H. An, L. Cheng, D. J. White, and T. Griffiths, "Stability of subsea pipelines during large storms," Philos. Trans. R. Soc. London, Ser. A 373(2033), 20140106 (2015).

${ }^{7}$ C. H. K. Williamson, "Vortex dynamics in the cylinder wake," Annu. Rev. Fluid Mech. 28(1), 477-539 (1996).

${ }^{8}$ H. An, L. Cheng, and M. Zhao, "Direct numerical simulation of oscillatory flow around a circular cylinder at low Keulegan-Carpenter number," J. Fluid Mech. 666, 77-103 (2011).

${ }^{9}$ F. Tong, L. Cheng, M. Zhao, T. Zhou, and X. B. Chen, "The vortex shedding around four circular cylinders in an in-line square configuration," Phys. Fluids 26(2), 024112 (2014).

${ }^{10} \mathrm{M}$. Breuer, J. Bernsdorf, T. Zeiser, and F. Durst, "Accurate computations of the laminar flow past a square cylinder based on two different methods: Lattice-Boltzmann and finite-volume," Int. J. Heat Fluid Flow 21(2), 186196 (2000).

${ }^{11} \mathrm{~K}$. Williamson, "The existence of two stages in the transition to threedimensionality of a cylinder wake." Phys. Fluids, 31(11), 3165-3168 (1988).

${ }^{12}$ M. Saeedi, P. P. LePoudre, and B. C. Wang, "Direct numerical simulation of turbulent wake behind a surface-mounted square cylinder," J. Fluids Struct. 51, 20-39 (2014).

${ }^{13}$ J. F. McClean and D. Sumner, "An experimental investigation of aspect ratio and incidence angle effects for the flow around surface-mounted finiteheight square prisms," J. Fluids Eng. 136(8), 081206 (2014).

${ }^{14}$ G. Palau-Salvador, T. Stoesser, J. Fröhlich, M. Kappler, and W. Rodi, "Large eddy simulations and experiments of flow around finite-height cylinders," Flow, Turbul. Combust. 84(2), 239-275 (2010).

${ }^{15}$ E. C. Joubert, T. M. Harms, and G. Venter, "Computational simulation of the turbulent flow around a surface mounted rectangular prism," J. Wind. Eng. Ind. Aerod. 142, 173-187 (2015).

${ }^{16}$ A. K. Saha, "Unsteady flow past a finite square cylinder mounted on a wall at low Reynolds number," Comput. Fluids 88, 599-615 (2013).

${ }^{17} \mathrm{C}$. W. Park and S. J. Lee, "Free end effects on the near wake flow structure behind a finite circular cylinder," J. Wind. Eng. Ind. Aerod. 88(2), 231-246 (2000).
${ }^{18}$ J. Y. Hwang and K. S. Yang, "Numerical study of vortical structures around a wall-mounted cubic obstacle in channel flow," Phys. Fluids 16(7), 23822394 (2004).

${ }^{19}$ V. Dousset and A. Pothérat, "Formation mechanism of hairpin vortices in the wake of a truncated square cylinder in a duct," J. Fluid Mech. 653, 519-536 (2010).

${ }^{20}$ A. Sau, R. R. Hwang, T. W. Sheu, and W. C. Yang, "Interaction of trailing vortices in the wake of a wall-mounted rectangular cylinder," Phys. Rev. E 68(5), 056303 (2003).

${ }^{21}$ D. Sumner, J. L. Heseltine, and O. J. P. Dansereau, "Wake structure of a finite circular cylinder of small aspect ratio," Exp. Fluids 37(5), 720-730 (2004).

${ }^{22}$ B. E. Lee, "The effect of turbulence on the surface pressure field of a square prism," J. Fluid Mech. 69(02), 263-282 (1975).

${ }^{23} \mathrm{H}$. Sakamoto and M. Arie, "Vortex shedding from a rectangular prism and a circular cylinder placed vertically in a turbulent boundary layer," J. Fluid Mech. 126, 147-165 (1983).

${ }^{24}$ S. Okamoto and Y. Sunabashiri, "Vortex shedding from a circular cylinder of finite length placed on a ground plane," J. Fluids Eng. 114(4), 512-521 (1992).

${ }^{25}$ H. F. Wang, Y. Zhou, C. K. Chan, and K. S. Lam, "Effect of initial conditions on interaction between a boundary layer and a wall-mounted finite-lengthcylinder wake," Phys. Fluids 18(6), 065106 (2006).

${ }^{26} \mathrm{~S}$. M. Hajimirzaie and J. H. Buchholz, "Flow dynamics in the wakes of low-aspect-ratio wall-mounted obstacles," Exp. Fluids 54(11), 1616 (2013).

${ }^{27}$ R. Porteous, D. J. Moreau, and C. J. Doolan, "A review of flow-induced noise from finite wall-mounted cylinders," J. Fluids Struct. 51, 240-254 (2014).

${ }^{28}$ T. Okamoto and M. Yagita, "The experimental investigation on the flow past a circular cylinder of finite length placed normal to the plane surface in a uniform stream," Bull. JSME 16(95), 805-814 (1973).

${ }^{29}$ H. Sakamoto and S. Oiwake, "Fluctuating forces on a rectangular prism and a circular cylinder placed vertically in a turbulent boundary layer," J. Fluids Eng. 106(2), 160-166 (1984).

${ }^{30}$ C. J. Baker, “The laminar horseshoe vortex," J. Fluid Mech. 95(02), 347-367 (1979)

${ }^{31}$ C. J. Baker, "The turbulent horseshoe vortex," J. Wind. Eng. Ind. Aerod. 6(1), 9-23 (1980).

${ }^{32}$ C. Lin, P. H. Chiu, and S. J. Shieh, "Characteristics of horseshoe vortex system near a vertical plate-base plate juncture," Exp. Therm. Fluid Sci. 27(1), 25-46 (2002).

${ }^{33}$ R. J. Pattenden, S. R. Turnock, and X. Zhang, "Measurements of the flow over a low-aspect-ratio cylinder mounted on a ground plane," Exp. Fluids 39(1), 10-21 (2005).

${ }^{34}$ C. V. Seal, C. R. Smith, O. Akin, and D. Rockwell, "Quantitative characteristics of a laminar, unsteady necklace vortex system at a rectangular block-flat plate juncture," J. Fluid Mech. 286, 117-135 (1995).

${ }^{35}$ A. S. W. Thomas, "The unsteady characteristics of laminar juncture flow," Phys. Fluids 30(2), 283-285 (1987).

${ }^{36}$ F. Ballio, C. Bettoni, and S. Franzetti, "A survey of time-averaged characteristics of laminar and turbulent horseshoe vortices," J. Fluids Eng. 120(2), 233-242 (1998).

${ }^{37}$ M. S. Adaramola, O. G. Akinlade, D. Sumner, D. J. Bergstrom, and A. J. Schenstead, "Turbulent wake of a finite circular cylinder of small aspect ratio," J. Fluids Struct. 22(6), 919-928 (2006).

${ }^{38}$ S. Roh and S. Park, "Vortical flow over the free end surface of a finite circular cylinder mounted on a flat plate," Exp. Fluids 34(1), 63-67(2003).

${ }^{39} \mathrm{C}$. W. Park and S. J. Lee, "Flow structure around a finite circular cylinder embedded in various atmospheric boundary layers," Fluid Dyn. Res. 30(4), 197-215 (2002).

${ }^{40}$ R. Hain, C. J. Kähler, and D. Michaelis, "Tomographic and time resolved PIV measurements on a finite cylinder mounted on a flat plate," Exp. Fluids 45(4), 715-724 (2008).

${ }^{41}$ A. Leder, "3D-flow structures behind truncated circular cylinders," in ASME/JSME 2003 4th Joint Fluids Summer Engineering Conference (American Society of Mechanical Engineers, 2003), pp. 825-831.

${ }^{42}$ C. R. Johnston and D. J. Wilson, "A vortex pair model for plume downwash into stack wakes," Atmos. Environ. 31(1), 13-20 (1997).

${ }^{43} \mathrm{D}$. Sumner, "Flow above the free end of a surface-mounted finite-height circular cylinder: A review," J. Fluids Struct. 43, 41-63 (2013).

${ }^{44} \mathrm{O}$. Frederich, E. Wassen, and F. Thiele, "Prediction of the flow around a short wall-mounted finite cylinder using LES and DES," J. Numer. Anal. Indust. Appl. Math. 3(3-4), 231-247 (2008). 
${ }^{45}$ J. A. Bourgeois, P. Sattari, and R. J. Martinuzzi, "Coherent vortical and straining structures in the finite wall-mounted square cylinder wake," Int. J. Heat Fluid Flow 35, 130-140 (2012).

${ }^{46} \mathrm{~S}$. Taniguchi, H. Sakamoto, and M. Arie, "Flow around circular cylinders of finite height placed vertically in turbulent boundary layers," Bull. JSME 24(187), 37-44 (1981).

${ }^{47}$ Z. Hosseini, J. A. Bourgeois, and R. J. Martinuzzi, "Large-scale structures in dipole and quadrupole wakes of a wall-mounted finite rectangular cylinder," Exp. Fluids 54(9), 1595 (2013).

${ }^{48}$ D. Zhang, C. Jiang, D. Liang, Z. Chen, Y. Yang, and Y. Shi, "A refined volume-of-fluid algorithm for capturing sharp fluid interfaces on arbitrary meshes," J. Comput. Phys. 274, 709-736 (2014).

${ }^{49}$ D. Zhang, C. Jiang, D. Liang, and L. Cheng, "A review on TVD schemes and a refined flux-limiter for steady-state calculations," J. Comput. Phys. 302, 114-154 (2015).
${ }^{50} \mathrm{O}$. Ubbink and R. I. Issa, "A method for capturing sharp fluid interfaces on arbitrary meshes," J. Comput. Phys. 153(1), 26-50 (1999).

${ }^{51}$ D. Zhang, C. Jiang, C. Yang, and Y. Yang, "Assessment of different reconstruction techniques for implementing the NVSF schemes on unstructured meshes," Int. J. Numer. Methods Fluids 74(3), 189-221 (2014).

${ }^{52}$ D. Zhang, C. Jiang, L. Cheng, and D. Liang, "A refined r-factor algorithm for TVD schemes on arbitrary unstructured meshes," Int. J. Numer. Methods Fluids 80(2), 105-139 (2016).

${ }^{53}$ A. Okajima, "Strouhal numbers of rectangular cylinders," J. Fluid Mech. 123, 379-398 (1982).

${ }^{54}$ K. M. Kelkar and S. V. Patankar, "Numerical prediction of vortex shedding behind a square cylinder," Int. J. Numer. Methods Fluids 14(3), 327-341 (1992).

${ }^{55}$ J. Jeong and F. Hussain, "On the identification of a vortex," J. Fluid Mech. 285, 69-94 (1995). 